ARTICLE

\title{
NRG1/ErbB signalling controls the dialogue between macrophages and neural crest-derived cells during zebrafish fin regeneration
}

Béryl Laplace-Builhé (i) 1, Audrey Barthelaix', Said Assou', Candice Bohaud', Marine Pratlong², Dany Severac², Gautier Tejedor (1) ${ }^{1}$, Patricia Luz-Crawford ${ }^{3,4}$, Mai Nguyen-Chi ${ }^{5}$, Marc Mathieu' ${ }^{1}$, Christian Jorgensen ${ }^{1,6} \&$ Farida Djouad ${ }^{1 凶}$

Fish species, such as zebrafish (Danio rerio), can regenerate their appendages after amputation through the formation of a heterogeneous cellular structure named blastema. Here, by combining live imaging of triple transgenic zebrafish embryos and single-cell RNA sequencing we established a detailed cell atlas of the regenerating caudal fin in zebrafish larvae. We confirmed the presence of macrophage subsets that govern zebrafish fin regeneration, and identified a foxd3-positive cell population within the regenerating fin. Genetic depletion of these foxd3-positive neural crest-derived cells ( $N C d C$ ) showed that they are involved in blastema formation and caudal fin regeneration. Finally, chemical inhibition and transcriptomic analysis demonstrated that these foxd3-positive cells regulate macrophage recruitment and polarization through the NRG1/ErbB pathway. Here, we show the diversity of the cells required for blastema formation, identify a discrete foxd3-positive $\mathrm{NCdC}$ population, and reveal the critical function of the NRG1/ErbB pathway in controlling the dialogue between macrophages and NCdC.

\footnotetext{
${ }^{1}$ IRMB, Univ Montpellier, INSERM, Montpellier, France. ${ }^{2}$ MGX, BCM, Univ Montpellier, CNRS, INSERM, Montpellier, France. ${ }^{3}$ Laboratorio de Inmunología Celular y Molecular, Facultad de Medicina, Universidad de los Andes, Santiago, Chile. ${ }^{4}$ IMPACT, Center of Interventional Medicine for Precision and Advanced Cellular Therapy, Santiago, Chile. ${ }^{5}$ LPHI, Univ Montpellier, CNRS, Montpellier, France. ${ }^{6} \mathrm{CHU}$ Montpellier, Montpellier, France.

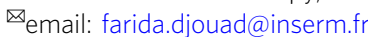


nlike adult mammals, urodele amphibians and fish species, such as zebrafish (Danio rerio), can regenerate entire parts of their body, including limbs and fins ${ }^{1,2}$, through a process referred to as epimorphic regeneration. This process involves the well-orchestrated restoration of multiple tissues and depends on the formation of a structure named blastema. Although for a long time, the blastema was considered a homogeneous mass of multipotent cells, it is now acknowledged that it is composed of heterogeneous, highly proliferative, and dynamic lineage-restricted progenitor cell types ${ }^{3-7}$. The list of cell types and factors involved in blastema formation and appendage regeneration has been expanded over the last years, but comparatively little is known about the cells and genes engaged upon tissue amputation in vertebrates.

To overcome this lack of knowledge, single-cell RNA sequencing (scRNA-seq) and lineage tracing experiments have been recently combined to study the axolotl limb blastema composition $^{8,9}$. These studies allowed clearly demonstrating that the heterogeneous fibroblast population of the blastema loses its adult features, adopts a multipotent skeletal progenitor phenotype, and expresses genes of the developmental-like state ${ }^{8,9}$. Furthermore, they confirmed the presence of muscle satellite cells, fibroblasts, and macrophages in the regenerating axolotl limbs, and also identified different cell types ${ }^{9}$. Altogether these studies revealed that single-cell transcriptome profiling provides a more detailed view of the blastema structure. It is now important to use this technology in the context of epimorphic regeneration in other vertebrates and at different developmental stages in order to find common features and understand how this regenerative outcome is regulated. This is especially of interest for developing innovative approaches for regenerative therapies. To this aim, a critical step forward would be to determine cell type-specific functions in the blastema of regenerating organisms.

Recently, a single-cell transcriptomic analysis performed at different time points during caudal fin regeneration in adult zebrafish revealed the cellular diversity of regenerating tissues and single-cell transcriptomic dynamics ${ }^{10}$. However, neither myeloid cells nor nerve or cells associated with nerves were identified in this study, despite their crucial role in epimorphic regeneration ${ }^{11-13}$. Specifically, in adult axolotls, macrophages are required for limb regeneration ${ }^{14,15}$. Similarly, in adult zebrafish, macrophage genetic depletion during the entire regeneration process impairs blastema cell proliferation and caudal fin regeneration, while their depletion during the tissue outgrowth phase affects the caudal fin morphology ${ }^{16}$. Using zebrafish larvae, we confirmed the presence of macrophages within the regenerating caudal fin fold and we observed an early and transient accumulation of pro-inflammatory macrophages ${ }^{17}$. Early recruited pro-inflammatory macrophages provides the accurate TNFa signal to prime blastema cell proliferation and regeneration in zebrafish ${ }^{17}$. However, the detailed cellular and molecular mechanisms responsible for macrophage recruitment and activation during epimorphic regeneration in zebrafish have not been elucidated yet. Moreover, Schwann cell (SC) precursors are neural crest (NC)-derived cells (NCdC) associated with almost all nerve fibers. These cells release trophic factors that promote blastemal cell proliferation and epimorphic regeneration ${ }^{18,19}$. In zebrafish, NCdC contribution has recently been demonstrated during cardiac development and regeneration ${ }^{20,21}$, but has never been investigated in appendage regeneration. Interestingly, after nerve injury, SC release factors that favour pro-inflammatory macrophage recruitment and polarization towards an anti-inflammatory phenotype $\mathrm{e}^{22,23}$. These studies suggest that SC might play a pivotal role in the tight regulation of the immune response required for regeneration.

Macrophage-mediated immune response and NCdC paracrine actions have been described separately in the context of regeneration. Therefore, there is a crucial need for a model of appendage regeneration in vertebrates that would integrate these two cell types to study their functional interactions.

In this work, scRNA-seq of cells from intact and regenerating caudal fin fold of zebrafish larvae allowed us to comprehensively describe the blastemal cell heterogeneity and generate an atlas of the cell types involved in regeneration. We identified cell populations within the blastema of regenerating zebrafish larvae, and demonstrated the presence of an orchestrator cell population that regulates the macrophage pro-regenerative response through the NRG1/ErbB signalling pathway. Collectively, these data have important implications for understanding epimorphic regeneration in vertebrates and for regenerative medicine.

\section{Results}

Identification of the different cell populations in the regenerating caudal fin fold by scRNA-seq. Caudal fin fold amputation in 3 days post fertilization ( $\mathrm{dpf}$ ) larvae induces a robust regeneration of the missing tissues within 3 days through the formation of the blastema at $24 \mathrm{~h}$ post-amputation (hpA). To determine the cellular and transcriptomic profiles of intact (uninjured) and regenerating caudal fin fold samples at $24 \mathrm{hpA}$, we used scRNAseq and the Chromium system (10X Genomics platform). This system employs the microdroplet technology to isolate individual cells, followed by next-generation sequencing, analysis and visualization of the single-cell datasets with the Cloud software (Fig. 1a). The two-dimensional (2D) distribution profiles obtained by Uniform Manifold Approximation and Projection (UMAP) revealed seven distinct cell clusters $(\mathrm{K}-\mathrm{mean}=7)$ in the regenerating fin fold (Fig. 1b). Then, we generated the lists of the top 100 upregulated transcripts (filtered by $\mathrm{FDR}<0.05$ and ranked by fold change) in each cluster of the regenerating fin sample (Fig. 1b and Supplementary Data 1). Hierarchical clustering based on these differentially expressed genes showed specific signatures in each cluster (Fig. 1c). We used well-structured lists of markers to assign a cell identity to each cluster. We assessed the robustness of these markers by reviewing the literature (Supplementary Data 2). In the regenerating fin sample, we focused on cluster 7 because it included cells that expressed neuron and glia markers, such as CD59, tfap2 and egr2 (Supplementary Data 1). In cells of this cluster, $h m \times 3 a$ (a gene encoding a protein predicted to have sequence-specific DNA binding activity) and $\mathrm{irg} 1 \mathrm{l}$ (a gene encoding a protein involved in the inflammatory response to wounding) also were upregulated, suggesting that this cell population might specifically respond to amputation (Fig. 1d-f). Moreover, in cluster 7, we identified cells that overexpressed foxd3, sox10, and $\operatorname{erg} 2 b$ (Fig. 1f), and the presence of a specific signature in $f \circ x d 3^{+}, \operatorname{sox} 10^{+}$, and $\operatorname{erg} 2 b^{+}$cells (Fig. 1g). We then confirmed, in cluster 6, the presence of myeloid cells that express card9, illb, and spia. Cluster 5 contained cells that strongly expressed $n r 2 f 1 a$ (involved in circulatory system development and endothelial cell proliferation) and genes involved in cell cycle and proliferation (aurkb, top2a, mki67). Also, we confirmed $f g f 8 a$ expression in apical epithelium cap cells (cluster 4), and highlighted the expression of matrix metalloproteinase-encoding genes ( $m m p 9$ and $m m p 13 a)$. Cluster 3 corresponded to mesenchymal cells and epidermis/mesenchymal cells and expressed mesenchymal cell markers ( $p d g f r l$, twist $1 b$, twist 3 ). Clusters 2 and 3 were also enriched in extracellular matrix protein-encoding genes (col5a2a, colla2a). In cluster 1 that included epidermis cells, cldne, evpla and agr 1 were strongly expressed. However, we could not formally identify cells in cluster 2 because they did not specifically express any typical cell/tissue marker. We hypothesized that this cluster comprised epidermis cells because they expressed lgals1l1 or krt91. 
a
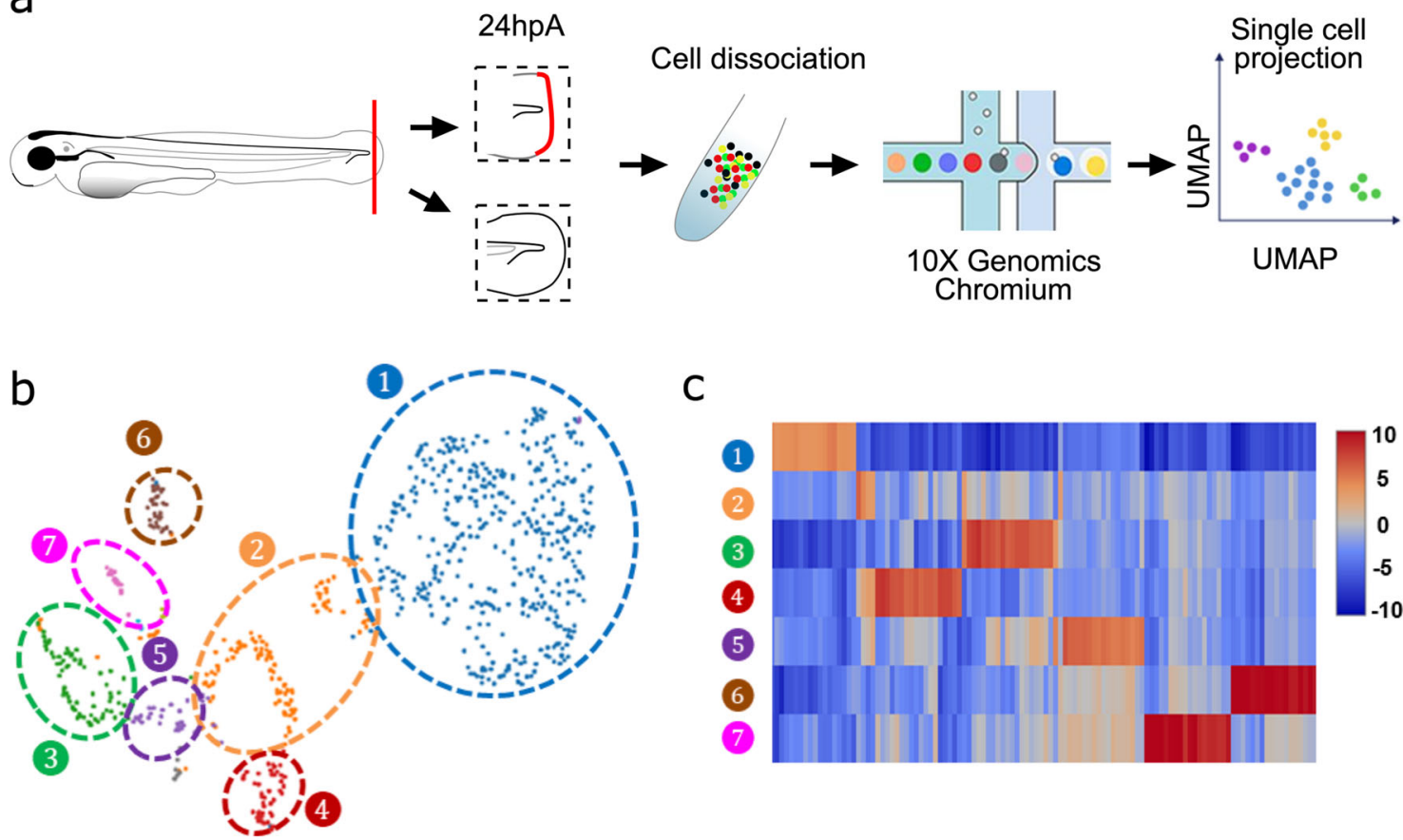

C

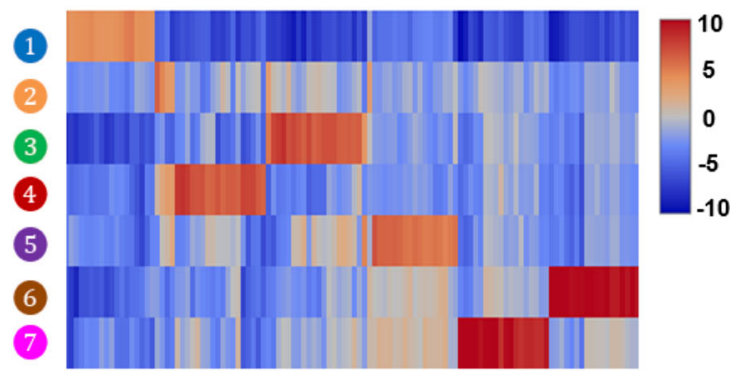

d

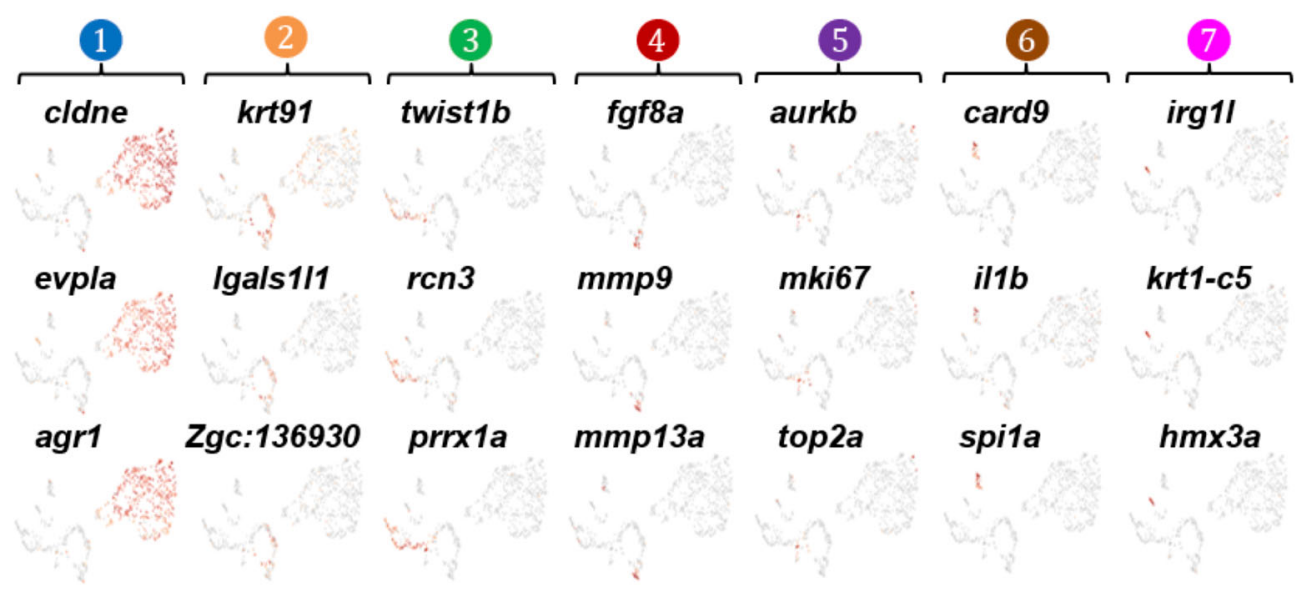

e
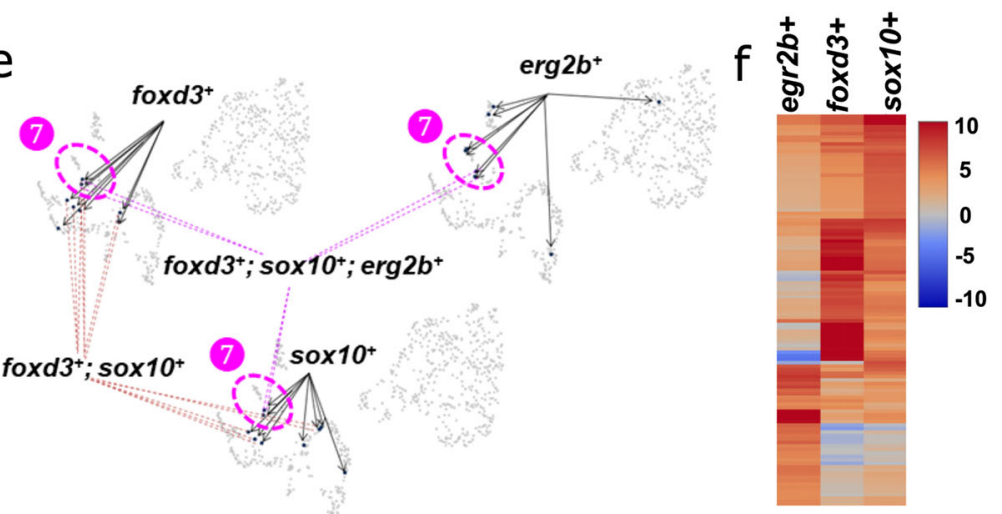

epidermis

cluster 2

mesenchyme

apical epithelial cap

mitotic cells

myeloid cells

neurons and glia

Fig. 1 Characterization of cells in the zebrafish blastema by scRNA-seq. a scRNA-seq experiment design. b Uniform Manifold Approximation and Projection (UMAP) plots visualizing RNA-sequencing data of single cells from the cut caudal fin fold. Seven different cell clusters were identified c Heatmap displaying the genes that are differentially expressed in the seven clusters. Red, upregulated genes, and blue, downregulated genes. d UMAP representation of the expression (low to high, grey to red) of cell markers in the different clusters. e Foxd3, sox10, and egr $2 b$ expression in cluster 7 (low to high expression, grey to blue). $\mathbf{f}$ Heatmap of the gene expression profile of the foxd $3^{+}$, sox $10^{+}$, and egr $2 b^{+}$cell populations. 
Moreover, we used the Cell Ranger software to aggregate and compare the expression data in the amputated fin and in the intact control (Supplementary Fig. 1a). The comprehensive list of the 100 differentially expressed genes is presented in Supplementary Data 3. Then, we used marker genes (Supplementary Data 2) and the UMAP method to distinguish cell subpopulations in the uninjured and injured samples. This analysis showed that cell clusters were quite comparable between conditions. This is in line with a similar study carried out in Xenopus laevis ${ }^{24}$ showing that wound epidermis is not a novel cell state, but a redeployment of the embryonic apical epidermal ridge. As our study relied on developing zebrafish larvae and on the basis of the similitudes between clusters of the two studies, we propose the same hypothesis. The most striking difference was the strong increase of the myeloid cell cluster in the injured condition compared with uninjured samples. This result was expected because myeloid cells are recruited after amputation (Supplementary Fig. 1c). Finally, analysis of the uninjured sample datasets (Supplementary Fig. 1d) revealed the presence of another glia/neuron subpopulation type in cluster 6 and some muscle markers in cluster 4 , indicating light contamination from the distal trunk muscle cells in this sample (Supplementary Fig. 1e-f). The lists of the top 100 upregulated transcripts in each cluster of the intact fin sample are detailed in Supplementary Data 4 . Altogether, these results demonstrated the cell type heterogeneity of the regenerating caudal fin fold of zebrafish larvae and identified a cell population that corresponds to $f \circ x d 3^{+}$sox $10^{+}$erg $2 b^{+} \mathrm{NCdC}$.

In the regenerating caudal fin fold, foxd $3^{+} \mathrm{NCdC}$ exhibit morphological and phenotypic changes. The scRNA-seq analysis revealed the existence of cells that express NC cell markers (cluster 7), including foxd3. NC cells express several transcription factors, such as foxd3 and sox10, that display pivotal functions during NC development ${ }^{24}$. Foxd 3 is expressed in pre-migratory NC cells, and also in some migrating and differentiating NC cells $^{25}$. In urodele amphibians, NCdC release trophic factors that promote blastemal cell proliferation and epimorphic regeneration $^{18}$. In zebrafish, NCdC contribute to cardiac development and regeneration ${ }^{20,21}$, but their role in appendage regeneration is not known. To determine whether the foxd $3^{+}$cell population in the blastema was involved in caudal fin fold regeneration in zebrafish larvae, we first analysed foxd $3+$ NCdC presence and behaviour by confocal microscopy. First, analysis of $T g$ (foxd3:eGFP-F) zebrafish larvae showed the presence of $e G F P^{+}$ cells in the developing fin bud at the 11- and 14-somite stages (Fig. 2a) and in the caudal fin fold at day 3 post-fertilization (Fig. 2b, c). Second, to determine the relative proportion of foxd $3^{+}$cells in the intact fin fold mesenchyme at $3 \mathrm{dpf}$ and during regeneration (Fig. 2d), we used the triple transgenic zebrafish larvae $T g$ (foxd3:eGFP-F/rcn3:Gal4/UAS:mCherry) to track NCdC (green) and mesenchymal cells (red) ${ }^{12,26}$. Confocal microscopy imaging showed the presence of foxd3 $3^{+} \mathrm{NCdC}$ in the caudal fin fold mesenchyme at $3 \mathrm{dpf}$ (Fig. 2e; Supplementary Fig. 2a) and the increased number of foxd $3^{+} r c n 3^{+}$mesenchymal cells (yellow cells) at $6 \mathrm{hpA}$ and $24 \mathrm{hpA}$ in the caudal fin fold (Fig. 2e, Supplementary Fig. 2c, d). This observation could indicate the presence of an NCdC population that expresses mesenchymal cell markers within the regenerating fin, suggesting NCdC reprogramming or differentiation into mesenchymal cells during regeneration. FACS analysis of the regenerating caudal fin fold at 6 and 24 hpA confirmed this hypothesis (Fig. $2 \mathrm{f}-\mathrm{i}$ ). The percentage of foxd $3^{+} \mathrm{NCdC}$ was not significantly different in the intact and regenerating caudal fin fold at 6 and $24 \mathrm{hpA}$ (Fig. 2h). Conversely, the percentage of $m$ Cherry $^{+}$mesenchymal cells (Fig. $2 \mathrm{~g}$ ) and of $m$ Cherry ${ }^{+}$eGFP ${ }^{+}$mesenchymal cells (Fig. 2i) was significantly higher in the regenerating caudal fin fold at $24 \mathrm{hpA}$. Finally, at $6 \mathrm{hpA}$, roundness (Supplementary Fig. 2e) and circularity (Supplementary Fig. 2f) of foxd $3^{+} r c n 3^{+}$cells were increased and the cell elongation factor (Supplementary Fig. 2g) was decreased at the wound site compared with control caudal fin fold (uncut). Altogether, our results suggest that these morphological changes accompany the phenotypic and functional modifications of $f \circ x d 3^{+}$cells during regeneration.

In the blastema, mesenchymal cells proliferate in contact with foxd $3^{+} \mathrm{NCdC}$. To better understand the increase of foxd3 ${ }^{+} \mathrm{rcn}^{+}$ and $\mathrm{rcn}^{+}$cells upon caudal fin fold amputation, we assessed the proliferation rate of mesenchymal and non-mesenchymal cell populations throughout regeneration in $\mathrm{Tg}(\mathrm{rcn} 3:$ Gal4/UAS:mCherry) and $T g$ (foxd3:eGFP-F) larvae by immunodetection of phosphorylated histone 3 ( $\mathrm{PH} 3)$ that labels proliferative cells. At the wound site, the proliferation rate of $\mathrm{PH}^{+}{ }^{+} \mathrm{rnn} 3^{+}$mesenchymal cells was significantly higher than that of $\mathrm{PH}^{+}{ }^{+} \mathrm{cn} 3^{-}$cells at 6 and 24 hpA (Fig. 3a). Conversely, upon amputation, foxd $3^{+}$ cells did not proliferate (Fig. 3a and Supplementary Fig. 3a). Moreover, all proliferating $\mathrm{rcn}^{+}$cells were physically close to foxd $3^{+} \mathrm{NCdC}$, as revealed by $4 \mathrm{D}$ confocal microscopy at $6 \mathrm{hpA}$ in Tg(foxd3:eGFP-F/rcn3:Gal4/UAS:mCherry) larvae (Fig. $3 \mathrm{~b}$ and Movie 1). This observation was confirmed by counting $\mathrm{PH}^{+}$ cells in the contact area with foxd $3^{+}$cells (Supplementary Fig. 3bc). Indeed, the percentage of $\mathrm{PH}_{3}{ }^{+}$cells in contact with foxd3 $3^{+}$ cells was higher than that of $\mathrm{PH}^{+}$cells alone in the cut condition (Supplementary Fig. 3b), but not in the uncut condition (Supplementary Fig. 3c). These results confirmed the presence of fox $3^{+} \mathrm{NCdC}$ in the regenerating caudal fin fold, and identified phenotypic and morphological changes in caudal fin fold mesenchymal cells during regeneration. Moreover, some of this newly identified foxd $3^{+} \mathrm{NCdC}$ interact tightly with proliferating mesenchymal cells.

Foxd $3^{+}$NCdC are required for zebrafish caudal fin fold regeneration. The observed morphologic and phenotypic changes in $f_{0 x d}{ }^{+} \mathrm{NCdC}$ and their physical interactions with proliferative mesenchymal cells during blastema formation suggested that foxd $3^{+} \mathrm{NCdC}$ could play a role in blastema cell proliferation and formation during caudal fin fold regeneration. To address this hypothesis, we partially depleted foxd $3^{+} \mathrm{NCdC}$ using morpholino-mediated knockdown of foxd $3^{27}$. Foxd 3 governs the expression of other critical transcription factors, such as snail and sox10, in nascent NC cells and promotes NC cell subset survival $^{28}$. Moreover, the zebrafish sym 1 mutant, which possesses a functional null allele of the zebrafish foxd3 gene, exhibits a normal number of pre-migratory NC cells, but a reduced number of cells that delaminate from the neural tube, and a defect in snail and sox10 expression levels ${ }^{29}$. The disappearance of NCderivative structures, such jaw and otic vesicle, and the reduced number of pigment cells that appeared immature in foxd3 morphants (MOfoxd3) compared with control morphants (MOctl) (Supplementary Fig. 3d) confirmed the loss of Foxd3 activity. Moreover, we used the $\mathrm{Tg}(\text { foxd3:mCherry })^{c t 110}$ heterozygote line that expresses the FoxD3-mCherry fusion protein ${ }^{30}$ to validate the efficacy of the foxd3 morpholino (MOfoxd3) (Supplementary Fig. 3e). Confocal microscopy showed that, in $3 \mathrm{dpf}$ embryos injected with $\mathrm{MOfoxd} 3$, the number of foxd $3^{+} \mathrm{NCdC}$ within the mesenchyme was reduced (Fig. 3c). Caudal fin fold regeneration involves the critical step of blastemal cell proliferation in the area beneath the apical epithelial cap (AEC) from $6 \mathrm{hpA}$, followed by cell proliferation propagation to more proximal regions from $24 \mathrm{hpA}^{31}$. Analysis of cell proliferation by $\mathrm{PH} 3$ immunodetection in the $24 \mathrm{hpA}$ blastema of MOctl- and MOfoxd3-injected 


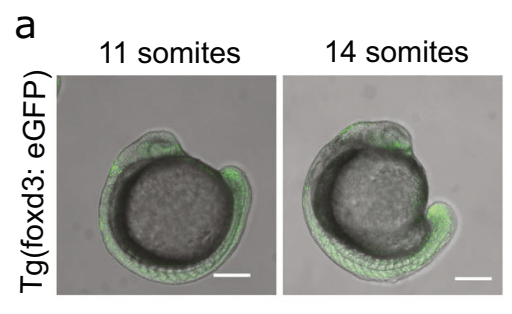

b
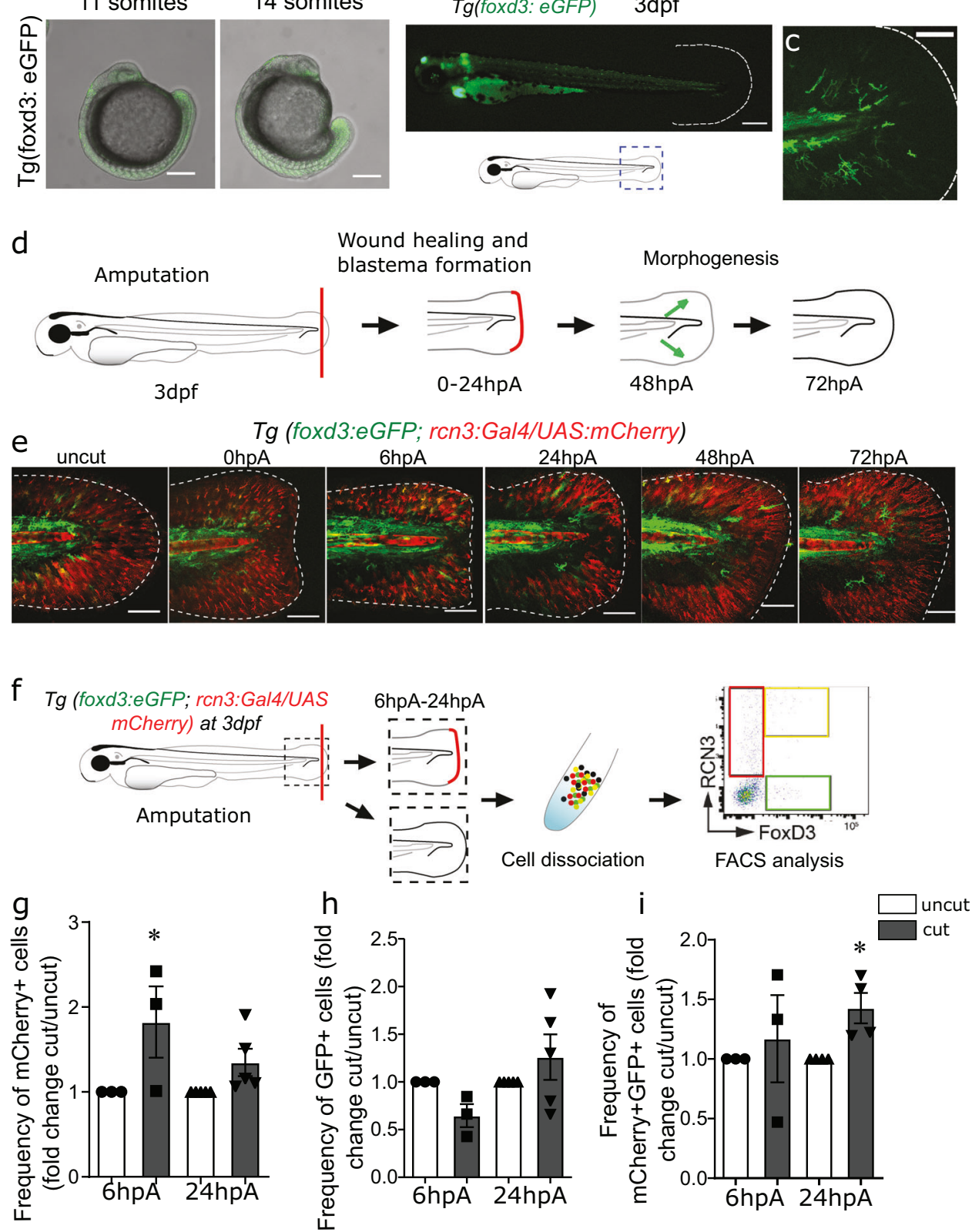

Fig. 2 Foxd3 ${ }^{+} \mathrm{NCdC}$ in the developing and regenerating caudal fin fold mesenchyme are located next to proliferative $r c n 3^{+}$mesenchymal cells.

a Confocal images of $T g$ (foxd3:eGFP-F) zebrafish embryos at 11 somites and 14 somites (Scale bars $=300 \mu m$ ). $\mathbf{b}$, c Representative fluorescent images of $\mathrm{Tg}$ (foxd3:eGFP-F) $3 \mathrm{dpf}$ larvae (Scale bar $=400 \mu \mathrm{m}$. (b) scale bar $=80 \mu \mathrm{m}$ (c) d Schematic representation of caudal fin fold regeneration after amputation of $3 \mathrm{dpf}$ larvae. e Representative confocal microscopy images of $T g$ (foxd3:eGFP-F/rcn3:Gal4/UAS:mCherry) larvae during regeneration (Scale bars $=80 \mu \mathrm{m}$, representative from 5 biologically independent larvae examined over 3 independent experiments). $\mathbf{f}$ Schematic representation of the fluorescent-activated flow cytometry analysis of eGFP+ and mCherry ${ }^{+}$cells from caudal fin fold of $T g$ (foxd3:eGFP-F/rcn3:Gal4/UAS:mCherry) larvae at 6 and 24 hpA. Data were analysed using FlowJo v10. g Frequency of $m$ Cherry ${ }^{+}$cell number in the caudal fin fold at 6 and 24 hpA relative to the age-matched uninjured controls. h Frequency of eGFP+ cell number in the caudal fin fold at 6 and $24 \mathrm{hpA}$ relative to the age-matched uninjured controls (fold change). i Frequency of mCherry ${ }^{+}$GFP $^{+}$cell number in the caudal fin fold at 6 and $24 \mathrm{hpA}$ relative to the age-matched uninjured controls. g-i Graphs represent the mean value \pm SEM, one-tailed Wilcoxon test was performed, ${ }^{\star} p<0.05, p=0.05(\mathbf{g}), p=0,0143(\mathbf{i}), n=50-300$ larvae per group from 5 independent experiments.

zebrafish larvae revealed that cell proliferation rate was significantly lower in regenerating foxd3 morphants (MOfoxd3) compared with controls (MOctl) (Fig. 3d) and consequently caudal fin fold growth was significantly reduced, compared with embryos injected with control morpholino (MOctl) (Fig. 3e). This was correlated with the altered expression pattern of junbl, a blastemal marker ${ }^{31}$, observed by in situ hybridization (Supplementary Fig. 3f). To confirm NCdC role in blastemal cell proliferation, we used homozygous $T g($ foxd3:mCherry)ct110 $3 \mathrm{dpf}$ larvae that present similar defects in NC cell development as foxd 3 morphants ${ }^{30}$. Compared with control zebrafish larvae (wild type, WT), fin fold outgrowth was severely impaired in Tg(foxd3:mCherry $)^{c t 110}$ mutant larvae at $72 \mathrm{hpA}$ (Fig. 3h). This impairment of the regenerative capacity was associated with a significant decrease of $\mathrm{PH}^{+}$cells in the blastema compared with WT zebrafish (Fig. $3 \mathrm{~g}$ ), as well as a decrease of junbl expression at 


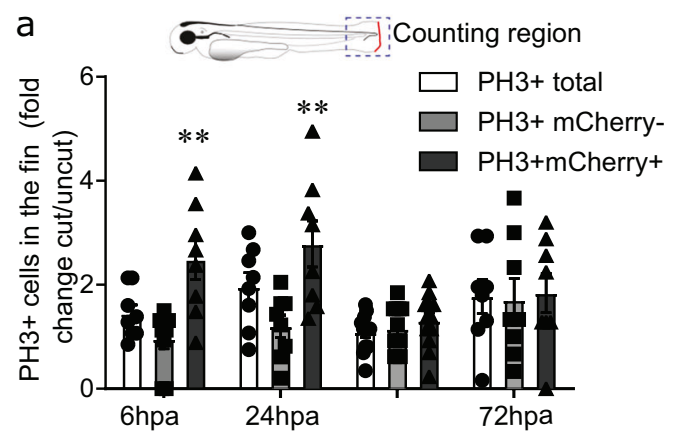

b

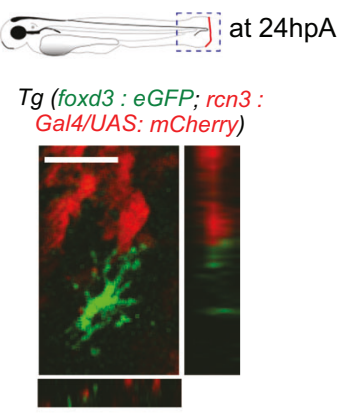

C
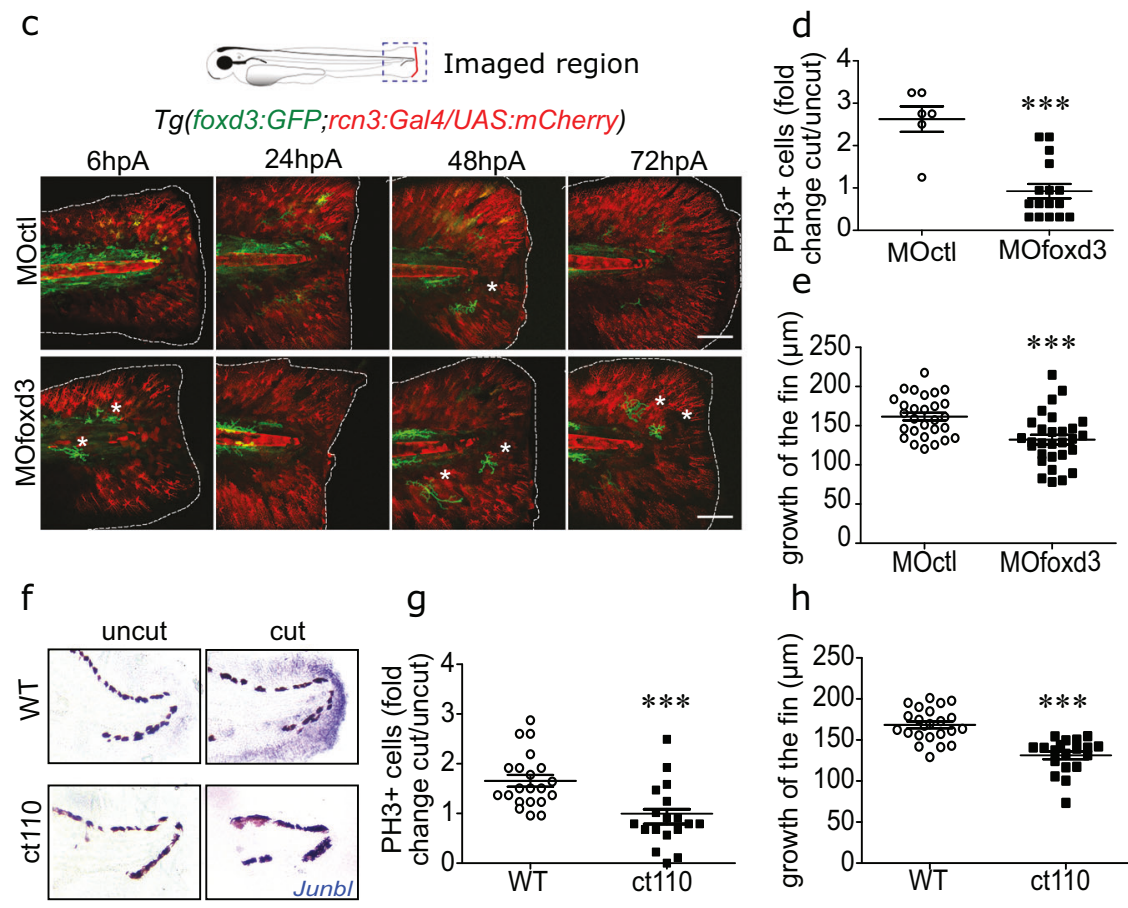

g

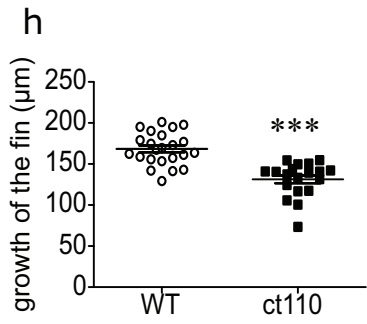

Fig. 3 Foxd3 + NCdC are required for blastemal cell proliferation and zebrafish caudal fin fold regeneration. a Quantification of cell proliferation in the blastema of $\mathrm{Tg}$ (rcn3:Gal4/UAS:mCherry) larvae from $6 \mathrm{hpA}$ to $72 \mathrm{hpA}$. Mitotic cells were detected using an anti-PH3 antibody. Data are shown as fold change relative to the age-matched uninjured controls, and are the mean $\pm S E M, n=8$ (for the $6,24,72 \mathrm{hpA}$ time points), $n=12$ (for the 48 hpA time point), ${ }^{\star \star} p<0.01$. b Image of a time-lapse $z$-stack sequence of a Tg(foxd3:eGFP-F/rcn3:Gal4/UAS:mCherry) larvae at $24 \mathrm{hpA}$ with $\mathrm{x}$ and y projections using the Fiji software (Scale bar $=8 \mu \mathrm{m}$ ). c Confocal images of caudal fin fold of Tg(foxd3:eGFP-F/rcn3:Gal4/UAS:mCherry) larvae injected with MOctl or MOfoxd3 at $3 \mathrm{dpf}$, and then from $6 \mathrm{hpA}$ to $72 \mathrm{hpA}$ (asterisks show pigments, scale bars $=80 \mu \mathrm{m}$, representative of $n=5$ larvae from 3 independent experiments). d Blastemal cell proliferation in foxd3 and control morphants was assessed using an anti-PH3 antibody at 24 hpA. Data are shown as fold change relative to the age-matched uninjured controls, and error bars are the SEM, $n=6$ (for the control group) and $n=16$ (for the foxd3 morphants) biologically independent larvae, one-tailed Mann-Whitney test was performed, $p=0.0007,{ }^{\star \star \star} p<0.001$. e Quantification of caudal fin fold length in foxd3 and control morphants at $72 \mathrm{hpA}$. Error bars are the SEM, $n=21$ biologically independent larvae, one-tailed Mann-Whitney test was performed, $p=0.0002,{ }^{\star \star \star} p<0.001$. f The mRNA expression of the blastemal marker junb-l (blue) was detected by in situ hybridization at 24 hpA in control and amputated fin folds from $4 \mathrm{dpf}$ wild type (WT) and Tg(foxd3:mCherry)ct110 (ct110) mutant larvae (representative of $n=15$ biologically independent larvae per groups). $\mathbf{g}$ Blastemal cell proliferation in heterozygotes (WT) and homozygotes (ct110) $T g$ (foxd3:mCherry) ct110 larvae at 24 hpA was detected using an anti-PH3 antibody (graph represents the mean number of positive cells \pm SEM, $n=21$ (for the WT groups) and $n=18$ (for mutants) larvae from 3 independent experiments, one-tailed Mann-Whitney test was performed, $p=0.0002,{ }^{\star \star \star} p<0.001$ ). (h) Quantification of fin fold growth at $72 \mathrm{hpA}$ in WT and $\mathrm{Tg}$ (foxd3:mCherry) ct170 mutant $6 \mathrm{dpf}$ larvae (graph represents the mean \pm SEM, $n=22$ (for the WT group), $n=19$ (for the mutant group) biologically independent larvae, one-tailed Mann Whitney test was performed, $\left.p=0.0001,{ }^{\star \star \star} p<0.001\right)$.

$24 \mathrm{hpA}$ (Fig. 3f). Altogether, these data revealed that NCdC are required for successful caudal fin fold regeneration, partly by inducing blastema cell proliferation and thereby blastema formation.

Foxd3 $^{+}$NCdC induce macrophage recruitment and activation during regeneration. Macrophage subpopulations are essential for appendage regeneration in zebrafish ${ }^{11}$ and the balance between M1- and M2-like macrophages is crucial for providing the tightly regulated TNFa signal and inducing regeneration ${ }^{12}$. In Wallerian degeneration, SC, NCdC exert several functions allowing nerve regeneration, release some factors essential for macrophage recruitment. Particularly, it has been suggested that SC promote the recruitment of pro-inflammatory macrophages and induce their polarization toward an anti-inflammatory phenotype $^{23}$. Therefore, we investigated the role of the foxd $3^{+} \mathrm{NCdC}$ in macrophage recruitment and polarization during epimorphic regeneration. First, using Tg(mpeg1:mCherry- 
a
Imaged region at $\mathrm{OhpA}, 6 \mathrm{hpA}$,
Contacts assessed during 12 hours in $24 \mathrm{hpA}$
"tips" and "sides" zones

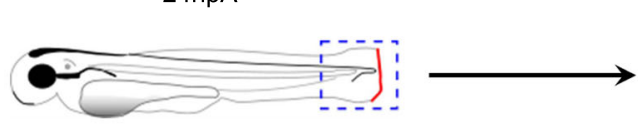

Tg(mpeg:mCherry-F; tnfa:eGFP; foxd3:eGFP)

b

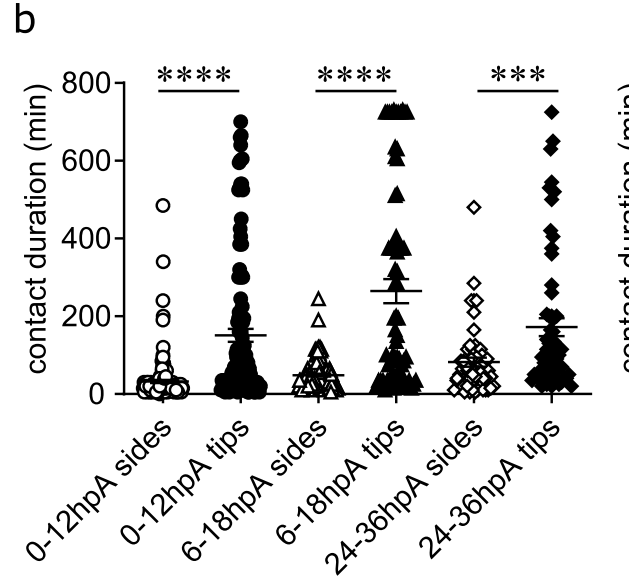

d
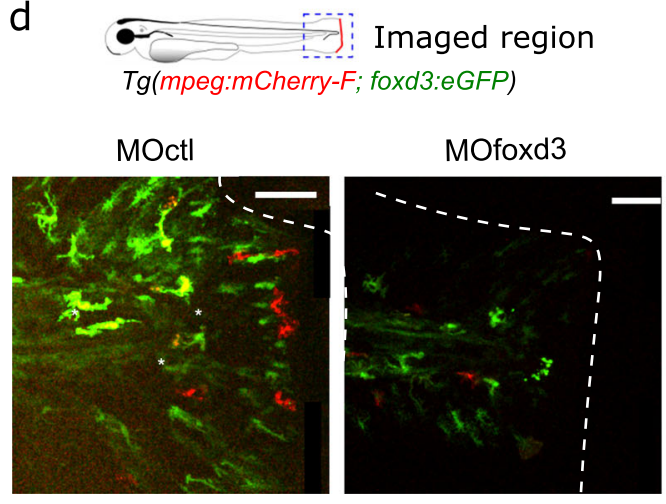

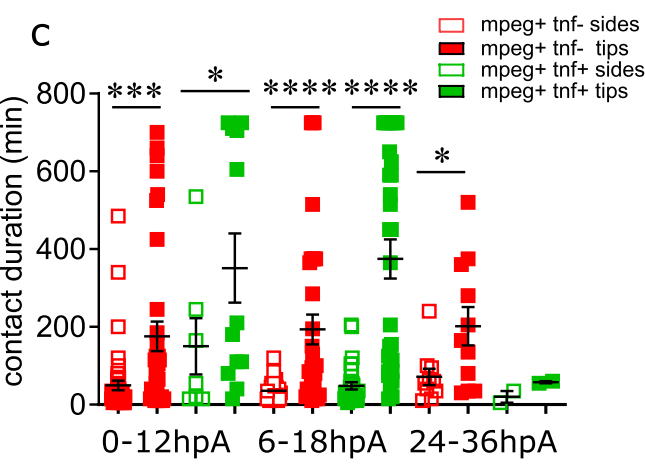
Tg(mpeg:mCherry-F; tnfa:eGFP; foxd3:eGFP)

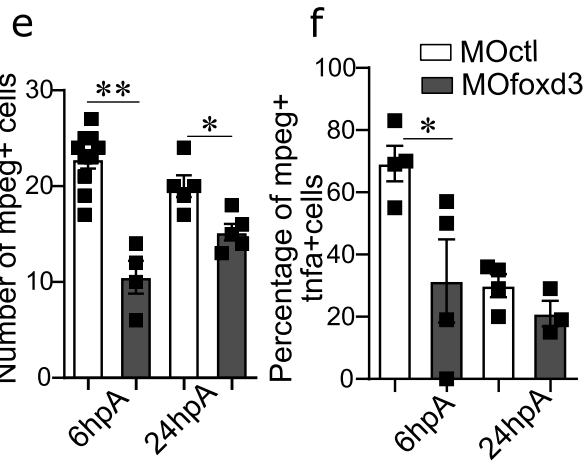

Fig. 4 Foxd3 ${ }^{+}$NCdC interact with and promote the recruitment and activation of macrophages in the blastema. a Schematic representation of the tip and sides where macrophages and foxd $3^{+} \mathrm{NCdC}$ contacts were assessed for $12 \mathrm{~h}$ by live confocal microscopy at $0 \mathrm{hpA}, 6 \mathrm{hpA}, 24 \mathrm{hpA}$ in Tg(mpeg:mCherryF; tnfa:eGFP-F; foxd3:eGFP-F) larvae. b Quantification of the duration of macrophages and foxd3 ${ }^{+} \mathrm{NCdC}$ contacts in the tips and sides during $12 \mathrm{~h}$ in Tg (mpeg:mCherry-F; tnfa:eGFP-F; foxd3:eGFP-F) larvae, at OhpA ( $n=192$ for the sides group, $n=124$ for the tips group), 6 hpA ( $n=105$ for the sides group, $n=71$ for the tips group), and $24 \mathrm{hpA}$ ( $n=54$ for the sides group, $n=60$ for the tips group) (mean number of contact durations \pm SEM, from at least $n=5$ biologically independent larvae per time points, Kruskal Wallis test with Dunn's test for multiple comparison were performed, ${ }^{\star \star \star *} p<0.0001$ ). c Quantification of the contact duration between macrophages (mpeg + and tnfa ${ }^{+}$) and foxd $3^{+}$cells in tips and sides during $12 \mathrm{~h}$ in the transgenic larvae, at $0 \mathrm{hpA}, 6 \mathrm{hpA}, 24 \mathrm{hpA}$ (mean number of contact durations \pm SEM, Kruskal-Wallis test and Dunn's test for multiple comparison were performed, ${ }^{*} p<0.05$, ${ }^{\star \star \star} p<0.001,{ }^{\star \star \star \star} p<0.0001, n=3$ biologically independent larvae per time point). d Fluorescence microscopy images of $3 \mathrm{dpf} T g$ (mpeg1:mCherry- $F /$ tnfa:eGFP-F/foxd3:eGFP-F) larvae injected with MOctl or MOfoxd3 (Scales bars $=50 \mu \mathrm{m}$ ). e Number of macrophages recruited at the wound site at $6 \mathrm{hpA}$ and $24 \mathrm{hpA}$ in MOctl- and MOfoxd3-injected Tg(mpeg1:mCherry-F) larvae (graph represents mean number of positive cells \pm SEM, $n=10$ biologically independent larvae for the control group at $6 \mathrm{hpA}, n=4$ larvae for the MOfoxd3 group at $6 \mathrm{hpA}, n=5$ larvae for both control and morphant group at $24 \mathrm{hpA}$, one-tailed Mann-Whitney test was performed, $p=0.0028$ for the $6 \mathrm{hpA}$ time point, $p=0.0106$ for the $24 \mathrm{hpA}$ time point, ${ }^{\star} p<0.05,{ }^{\star \star} p<0.01$ ). f Percentage of pro-inflammatory macrophages at the wound site at $6 \mathrm{hpA}$ and $24 \mathrm{hpA}$ (graph represents mean number of $m p e g^{+}$tnfa ${ }^{+}$cells \pm SEM, $n=4$ biologically independent larvae per groups' one-tailed Mann-Whitney test was performed, $p=0.0286$ for the 6 hpA time point, ${ }^{\star} p<0.05$ ).

F/tnfa:eGFP-F/foxd3:eGFP-F) $3 \mathrm{dpf}$ larvae, we monitored the interactions between foxd $3^{+} \mathrm{NCdC}$ and macrophage subpopulations at different time points during regeneration $(0,6,24 \mathrm{hpA})$ using confocal microscopy ( 12 hours of live imaging), and quantified the interactions between these cell types in two different regions of the fin fold (tip and sides) (Fig. 4a). We found that macrophages and foxd $3^{+} \mathrm{NCdC}$ had many contacts, and that long-lasting contacts occurred preferentially in the tip region (Fig. 4b). Moreover, the long-lasting contacts in the fin fold tips were more frequent between foxd $3^{+} \mathrm{NCdC}$ and $\operatorname{tnfa}{ }^{+}$macrophages (Fig. 4c, Movie 2). This result showed that contact frequency and duration between macrophages and foxd $3^{+} \mathrm{NCdC}$ depend on the region of the regenerating blastema. The finding that long-lasting contacts were preferentially made by pro-inflammatory 
$\left(m p e g^{+} \operatorname{tnfa} a^{+}\right)$macrophages suggests a possible role of foxd $3^{+}$ NCdC in their activation/polarization. To address this hypothesis, we injected MOfoxd3 in Tg(mpeg1:mCherry-F/tnfa:eGFP-F/fox$d 3: e G F P-F)$ embryos. Confocal microscopy showed the massive decrease of foxd $3^{+} \mathrm{NCdC}$ concomitantly with a substantial reduction of macrophage frequency in the regenerating blastema of MOfoxd3 morphants (MOfoxd3) compared with control morphants (MOctl) (Fig. 4d, e). Similarly, we did not observe any increase in mpeg1 expression in $\mathrm{Tg}(\text { foxd3:mCherry })^{c t 110}$ mutants following fin fold ablation in contrast to wild-type larvae in which mpeg1 expression was significantly increased in the $24 \mathrm{hpA}$ regenerating fin fold (Supplementary Fig. 4a). Of note, confocal microscopy and FACS analysis showed that the overall number of macrophages was not changed in foxd 3 morphants compared with controls (Supplementary Fig. 4b-e). Conversely, the number of $\operatorname{tnfa}$ + macrophages was reduced in foxd 3 morphants compared with control morphants (Fig. 4f, Movies 3 and 4). These results indicated that foxd 3 silencing significantly impaired macrophage recruitment at the injured site and their activation toward a pro-inflammatory tnfa ${ }^{+}$phenotype. These observations are in accordance with our results showing that $f \circ x d 3^{+} \mathrm{NCdC}$ establish preferential contacts with pro-inflammatory $t n \mathrm{fa}^{+}$macrophages during regeneration, and strongly suggest a positive role for foxd $3^{+} \mathrm{NCdC}$ in macrophage recruitment and activation during caudal fin fold regeneration via a paracrine mechanism.

Nrg1 and ErbB family members are expressed in blastemal cells and are required for appendage regeneration. To identify the paracrine mechanism underlying foxd $3^{+} \mathrm{NCdC}$ role in the macrophage response during regeneration, we focused our attention on neuregulin 1 (NRG1), a critical factor for the development of NC cells and of some NCdC, including SC ${ }^{32}$ that promotes the proliferation of damaged tissue cells in various models and during regeneration ${ }^{33-35}$. To determine whether NRG1/ErbB was one of signalling pathways that promote blastemal cell proliferation and macrophage pro-regenerative response, we first assessed the expression level of $n r g 1$ and erbb family members in our model. In situ hybridization analysis of 4 dpf larvae showed that $n r g 1$ was upregulated in the regenerating blastema in larvae at $24 \mathrm{hpA}$ compared with the intact caudal fin fold of control larvae (Fig. 5a). Then, analysis of the expression profile of the $n r g 1$ splicing variants described in zebrafish (i.e., nrg1.001, $n r g 1.002$, $n r g 1.003$ and $n$ rg1.004; ZFIN.org) revealed that $n r g 1.001$ and $n r g 1.002$ transcripts were not detectable in both intact and amputated fin fold (at $24 \mathrm{hpA}$ ), whereas the expression of $n$ rg1.003 $(p=0.0571)$ and $n r g 1.004 \quad(p<0.05 \quad n r g 1)$ was increased (Fig. 5f). Next, we assessed the expression profile of erbb2 and erbb3 that have been described as critical factors for regeneration in zebrafish ${ }^{36}$. While erbb3 expression level was comparable in regenerating blastema at $24 \mathrm{hpA}$ and in controls (supplementary Fig. 5a), erbb2 was significantly increased in response to caudal fin fold amputation (Fig. 5g). Then, we investigated the role of the NRG1 signalling pathway by adding PD168393 and AG1478, two specific inhibitors of the NRG1/ ErbB pathway ${ }^{36,37}$, to the water of $3 \mathrm{dpf}$ larvae on amputation day and every day until $72 \mathrm{hpA}$ (Fig. 5b). Compared with DMSOtreated amputated larvae (controls) in which caudal fin fold was fully regenerated at $72 \mathrm{hpA}$, in PD168393 or AG1478-treated amputated larvae fin fold regeneration was significantly impaired (Fig. 5d) and blastemal cell proliferation at $24 \mathrm{hpA}$ was significantly reduced (Fig. $5 \mathrm{c}$ ). These results are in line with previous studies and show that NRG1/ErbB signalling is necessary for caudal fin fold regeneration. Then, to determine which blastema cells expressed $n r g 1$, we focused on NCdC and performed in situ hybridization in control and foxd 3 morphants at $24 \mathrm{hpA}$. Foxd 3 deficiency was associated with massive $n r g 1$ downregulation (Fig. 5e). Of note, the four zebrafish $n r g 1$ variants were not differentially expressed in response to amputation in $\mathrm{Tg}($ foxd $3: \mathrm{m}$ Cherry)ct110 mutant larvae (Fig. 5f-g). Moreover, nrg1.004 expression levels were comparable in non-amputated $\operatorname{Tg}(\text { foxd3:mCherry })^{c t 110}$ and wild-type larvae. In $\operatorname{Tg}($ foxd3:mCherry) $)^{c t 110}$ mutants, erbb2, and erbb3 expression levels were not different compared with those in WT larvae and in response to amputation (Fig. 5g and Supplementary Fig. 5a). These results suggest that the significant erbb2 and nrg1.004 upregulation observed in wild-type larvae upon amputation depends on foxd3, and that foxd3-dependent NRG1/ErbB2 signalling has an essential role in appendage regeneration, by highlighting the correlation between $n r g 1$ expression and presence of foxd $3^{+}$cells in the regenerating blastema.

The Nrg1/ErbB2 signalling pathway is part of the molecular dialogue between macrophages and foxd $3^{+}$neural crestderived cells during regeneration. As foxd3 has a role in NRG1/ErbB2 signalling during regeneration and mammalian macrophages express several NRG1 receptors and migrate in response to NRG1 in vitro ${ }^{38}$, we investigated whether fox $3^{+} \mathrm{NCdC}$ promoted macrophage recruitment and activation through the NRG1/ErbB2 signalling pathway during caudal fin fold regeneration. To this aim, we first treated $3 \mathrm{dpf}$ amputated zebrafish with AG1478, a specific ErbB inhibitor (Fig. 5h). In treated zebrafish larvae, the number of macrophages $\left(\mathrm{mpeg}^{+}\right.$ cells) was significantly decreased in the injured fin fold at 6 and $24 \mathrm{hpA}$ (Fig. 5i). Moreover, macrophage activation and polarization toward a pro-inflammatory phenotype were reduced at 6 $\mathrm{hpA}$, as indicated by the lower number of $\operatorname{tnfa} a^{+}$macrophages in the regenerating fin fold of treated larvae compared with DMSO controls (Fig. 5j). These results suggest that NRG1/ErbB2 is necessary for macrophage recruitment and polarization during caudal fin fold regeneration.

As macrophage response to NRG1 has never been described during zebrafish caudal fin fold regeneration, we assessed the expression profile of $n r g 1.004$, erbb2, and erbb3 in FACS-sorted macrophage populations (Fig. 6a) from $3 \mathrm{dpf} \operatorname{Tg}$ (mpeg1:mCherryF/tnfa:eGFP-F) zebrafish larvae at $6 \mathrm{hpA}$ and $24 \mathrm{hpA}$. Although $n$ rg1.004, erbb2, erbb3 were expressed in all sorted cell populations (Fig. 6b, c, d, e, f, g), nrg1.004 transcript level was particularly abundant in negative cells (i.e., non-macrophage cells) derived from amputated larvae at $6 \mathrm{hpA}$ and $24 \mathrm{hpA}$, compared with control. Erbb2 and erbb3 also were mostly expressed in negative cells, but in macrophage populations, erbb2 and $e r b b 3$ (to a lower extent) transcripts were enriched in $\operatorname{tnfa}{ }^{+}$ macrophages at $6 \mathrm{hpA}$, and also at $24 \mathrm{hpA}$. These data show that zebrafish macrophages express $n r g 1$, erbb2, and erbb3, and that $e r b b 2$ and $e r b b 3$ are upregulated in the $t n f a^{+}$pro-inflammatory macrophage subset upon caudal fin fold amputation. This specific expression in $\operatorname{tnfa}{ }^{+}$macrophages and their preferential longlasting contacts with foxd $3^{+} \mathrm{NCdC}$ (Fig. 4) strongly suggest that macrophage recruitment and polarization are regulated by foxd $3^{+}$ NCdC through the NRG1/ErbB signalling pathway.

\section{Discussion}

In this study, we provide a cell atlas of the regenerating zebrafish caudal fin fold using the high-dimensional scRNA-seq approach. We found that the regenerating zebrafish caudal fin fold comprises seven cell types and identified a cell population within the regenerating fin fold: foxd $3^{+} \mathrm{NCdC}$. We then demonstrated that foxd $3^{+} \mathrm{NCdC}$ are required for caudal fin fold regeneration and that they regulate macrophage recruitment and activation, a 
a

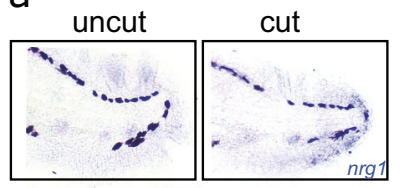

C
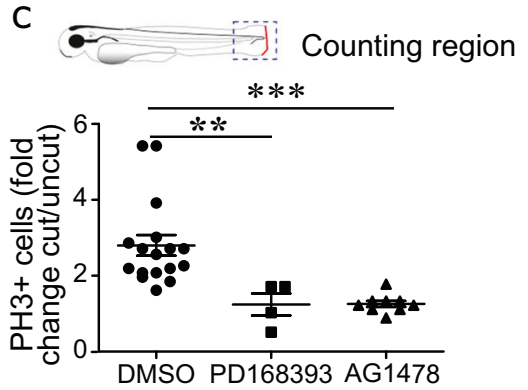

e

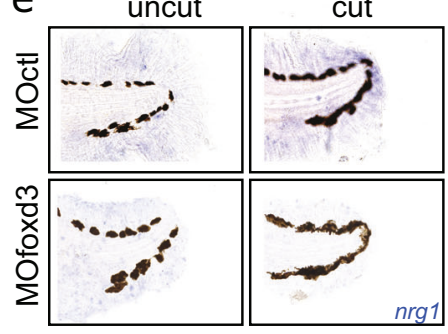

h Amputation Immersion with DMSO or

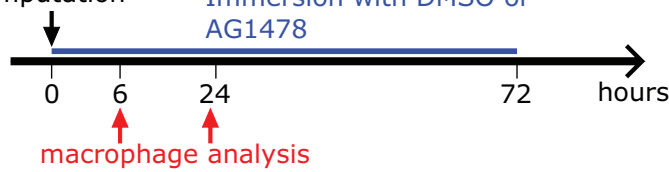

i
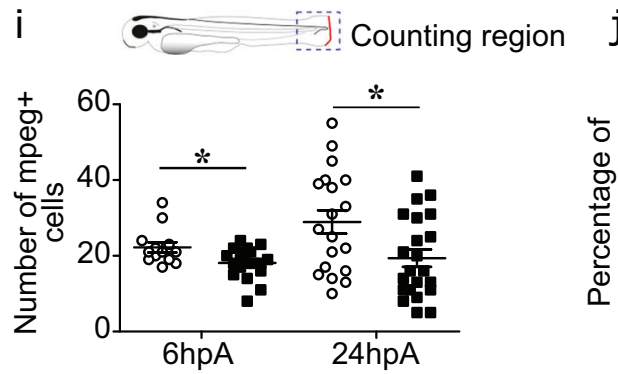

j b

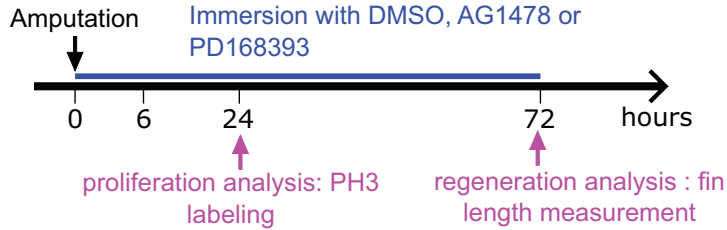

d

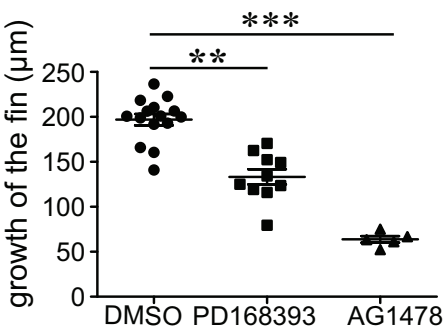

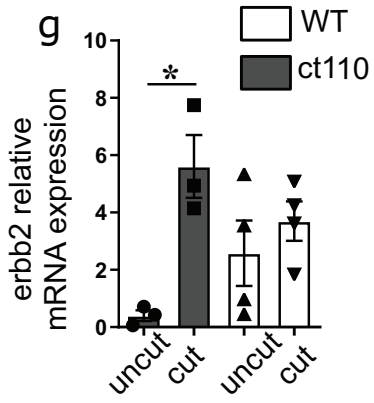

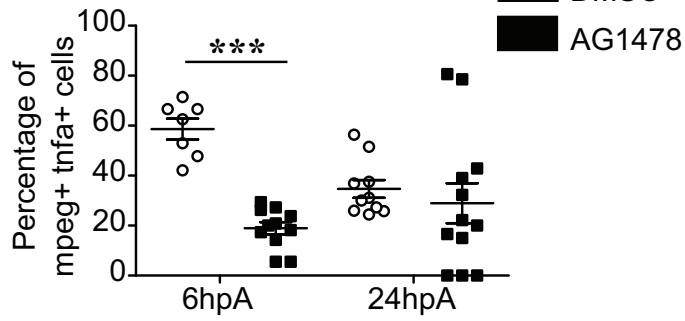

Fig. 5 Nrg1 and ErbB family members are expressed in blastemal cells and are required for appendage regeneration. a Representative image of nrg1 mRNA expression in uncut caudal fin fold and at $24 \mathrm{hpA}$ (from $n=15$ biologically independent larvae). $\mathbf{b}$ Wild type 3dpf larvae were amputated or not and exposed to DMSO, AG1478, or PD168393 for $72 \mathrm{~h}$. Cell proliferation and fin fold growth were measured at 24hpA and $72 \mathrm{hpA}$, respectively. c Quantification of cell proliferation in $24 \mathrm{hpA}$ larvae after the indicated treatments (graph represents means \pm SEM, $n=17$ larvae for DMSO group, $n=4$ larvae for PD168393 group, $n=9$ larvae for AG1478 group, Kruskal-Wallis test and Dunn's test for multiple comparisons were performed, ${ }^{\star \star} p<0.01$, ${ }^{\star \star \star} p<0.001$ ). d Quantification of fin fold growth in $72 \mathrm{hpA}$ wild type larvae after the indicated treatments (error bars show the SEM, $n=15$ larvae for DMSO group, $n=10$ larvae for PD168393 group, $n=5$ larvae for AG1478 group, Kruskal-Wallis test and Dunn's test for multiple comparisons were performed $\left.{ }^{*} p<0.01,{ }^{* \star *} p<0.001\right)$. e $n r g 1$ expression by in situ hybridization analysis in intact caudal fin folds and at $24 \mathrm{hpA}$ of MOfoxd3 or MOctl larvae (from $n=15$ biologically independent larvae). f Relative expression of $n r g 1.004$, and $\mathbf{g}$ erbb2 mRNA in wild type (WT) and Tg(foxd3:mCherry) ct110 mutant (ct110) larvae at $24 \mathrm{hpA}$ was assessed by RT-PCR using ef1a as reference gene (data are the mean \pm SEM, $n=15$ larvae per groups from 3 independent experiments, one-tailed Mann-Whitney test was performed, $\left.\mathbf{f} p=0.0286, \mathbf{g} p=0.05,{ }^{*} p<0.05\right)$. h 3dpf $\operatorname{Tg}$ (mpeg1:mCherry-F/tnfa:eGFP-F) larvae were amputated or not and exposed to DMSO or AG1478. Macrophage recruitment was analysed at $6 \mathrm{hpA}$ and $24 \mathrm{hpA}$. i Macrophage recruitment at the wound site in AG1478 or DMSO-treated Tg(mpeg1:mCherry-F) larvae at $6 \mathrm{hpA}$ and $24 \mathrm{hpA}$ (graph represents mean number of mCherry-positive cells \pm SEM, $n=13$ at $6 \mathrm{hpA}$ and $n=24$ at 24hpA for DMSO treated groups, $n=19$ at $6 \mathrm{hpA}$ and $n=22$ at $24 \mathrm{hpA}$ for AG1478 treated groups, one-tailed Mann-Whitney test was performed, $p=0.0154$ for the $6 \mathrm{hpA}$ time point, $p=0.0143$ for the $24 \mathrm{hpA}$ time point, ${ }^{*} p<0.05$ ). j Macrophage polarization at the wound site in AG1478 or DMSO-treated Tg(mpeg1:mCherry-F/tnfa:eGFP-F) larvae at 6 hpA and 24 hpA (graph represents mean number of mCherry+ eGFP ${ }^{+}$cells \pm SEM, $n=7$ at $6 \mathrm{hpA}$ and $n=10$ at $24 \mathrm{hpA}$ for DMSO treated groups, $n=11$ at $6 \mathrm{hpA}$ and $n=12$ at 24 hpA for AG1478 treated groups, onetailed Mann-Whitney test was performed, $p=0.0003$ for the $6 \mathrm{hpA}$ time point, ${ }^{\star \star \star} p<0.001$ ). 
a

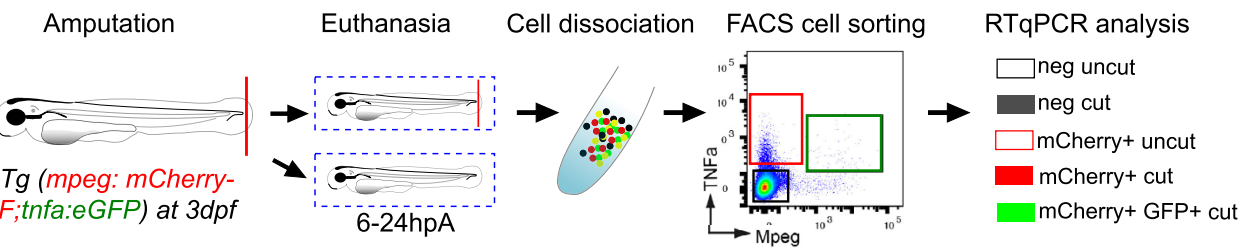

b
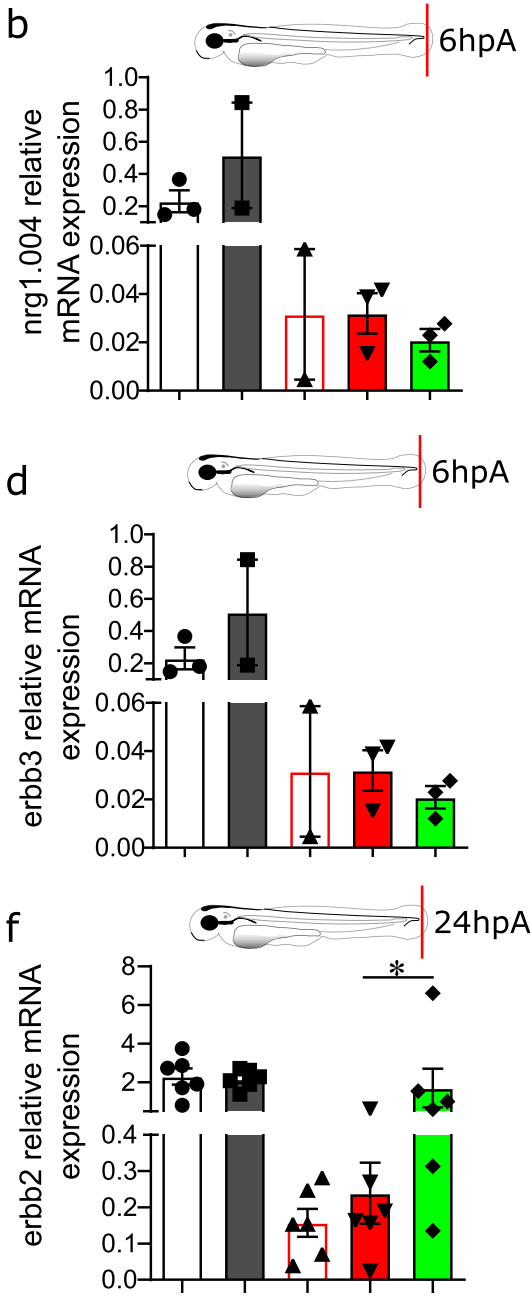

C
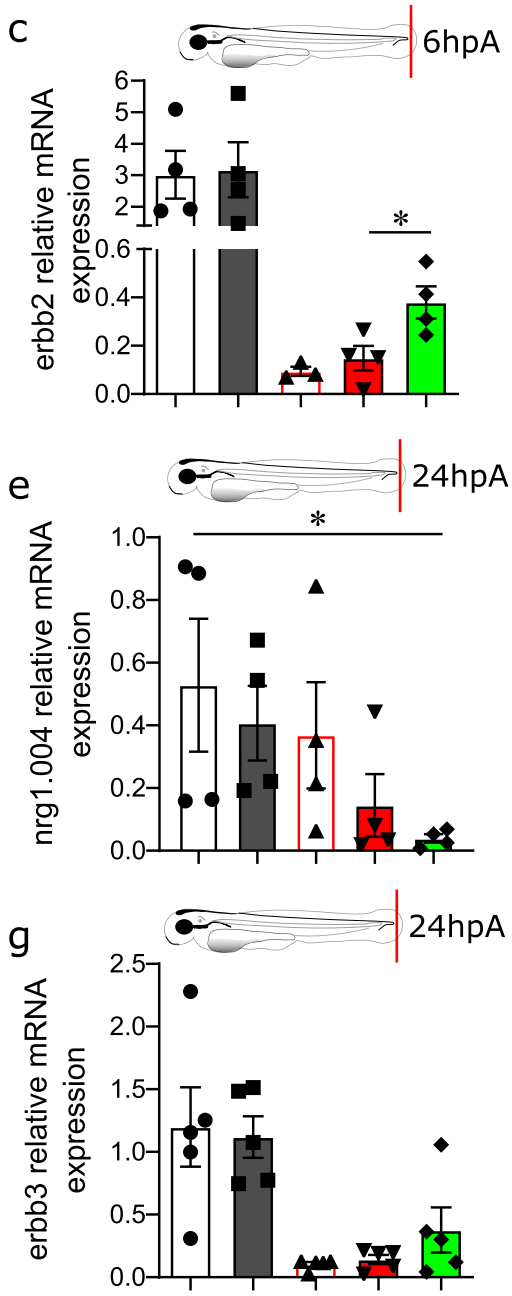

Fig. 6 Erbb2 is expressed by all blastemal cells including macrophages, and particularly pro-inflammatory macrophages. a $T g(m p e g 1: m C h e r r y-F /$ tnfa:eGFP-F) larvae were amputated or not (control) at $3 \mathrm{dpf}$, and cells were dissociated at 6 or $24 \mathrm{hpA}$ and sorted by FACS. Red, green, and black gates represent $m \mathrm{mCherry}^{+}, \mathrm{mCherry}{ }^{+} \mathrm{eGFP}{ }^{+}$, and $m \mathrm{mCherry}^{-} \mathrm{eGFP}{ }^{-}$cell populations, respectively. Relative expression of $\mathbf{b}, \mathbf{e} n r g 1, \mathbf{c}, \mathbf{f}$ erbb2, and $\mathbf{d}, \mathbf{g}$ erbb3 in $m C h e r r y$ ${ }_{-}^{-} \mathrm{eGFP}^{-}, \mathrm{mCherry}^{+}$and $m$ Cherry ${ }^{+} \mathrm{eGFP}+$ cells at $6 \mathrm{hpA} \mathbf{b}, \mathbf{c}, \mathbf{d}$ and $24 \mathrm{hpA} \mathbf{e}, \mathbf{f}, \mathbf{g}$ was quantified by RT-PCR on separated cells using ef1a as reference gene. b, $\mathbf{c}$, d Graphs represent the mean value \pm SEM, $n=200-300$ pooled larvae from 4 independent experiments; one-tailed Mann-Whitney test was performed, $p=0.0286,{ }^{\star} p<0.05$. e, $\mathbf{f}, \mathbf{g}$ Graphs represent the mean value \pm SEM, $n=200-300$ pooled larvae from 6 independent experiments; one-tailed Mann-Whitney test was performed, e $p=0.0143, \mathbf{f} p=0.0465,{ }^{\star} p<0.05$.

pivotal step in the regeneration process ${ }^{12}$, through the NRG1/ ErbB signalling pathway.

Our study provides a robust and efficient methodology to unbiasedly demonstrate the heterogeneity of the blastema formed after caudal fin fold amputation in zebrafish larvae. Live clonal analysis in zebrafish has allowed visualizing and quantifying, at high resolution, blastema formation, revealing the plasticity of blastema cells during regeneration. However, the exact timing of blastema formation and its heterogeneity were so far undefined. Here, we identified all the different cell types that compose the blastema, and also an orchestrator cell that governs the regeneration process. Indeed, our results show that foxd $3^{+} \mathrm{NCdC}$ drive the continuous regeneration of the injured tissue. Blastema formation, which is required for regeneration, is mediated through the release of trophic factors by NCdC in newts and mammals. For instance, production of the mitogenic Anterior Gradient protein (nAG) first by NCdC and then by the AEC is necessary and sufficient for blastemal cell proliferation and the rescue of the regeneration potential of denervated limbs ${ }^{18}$. Similarly, a recent study in mammals demonstrated that nerve-associated SC precursors dedifferentiate and secrete growth factors that promote blastema expansion and digit regeneration. Here, by combining the use of $4 \mathrm{D}$ confocal microscopy and transgenic larvae to track both $f_{0 x d 3^{+}} \mathrm{NCdC}$ and $\mathrm{rcn}^{+}$mesenchymal cells in the 
regenerating caudal fin fold from $6 \mathrm{hpA}$, we showed that all proliferating cells are mesenchymal cells that proliferate in contact with foxd $3^{+} \mathrm{NCdC}$. This observation highlights $\mathrm{NCdC}$ key role in blastema expansion and strongly suggests that blastemal cell proliferation is NCdC-dependent. This dependence was confirmed by the finding that $T g(\text { foxd3:mCherry })^{c t 110}$ mutants and foxd3 morphants cannot regenerate their fin fold after amputation. Altogether, these studies demonstrated that in regenerative species, appendage regeneration is possible through a conserved mechanism that relies on NCdC presence and activation. Of note, $\mathrm{NCdC}$ and other cell types that play an important role during appendage regeneration, such as neurons and endothelial cells, were not identified in the recent published study that described the cellular diversity of postinjury adult zebrafish fin ${ }^{10}$. This might be due to a systematic bias against some discrete cell subsets that might be generated during sample preparation or in the dropletbased scRNA-seq system. Increasing the number of cells or performing scRNA-seq of foxd3 $3^{+}$-sorted cells within the regenerating fin fold would help to identify this discrete cell subset and better study its role in the regeneration process of adult zebrafish fin.

The tight and long-lasting contacts between macrophages and foxd $3^{+}$NCdC suggested a functional interplay between these cell types, leading to the polarization toward a pro-inflammatory phenotype of the macrophages that interact with NCdC. Moreover, foxd3 silencing did not affect the total number of $m p e g 1^{+}$ macrophages in the entire zebrafish, but it significantly impaired their recruitment and activation at the injured site. Therefore, fox $3^{+}$NCdC are required for the generation of proinflammatory macrophages, presumably through direct contact, leading to the production of TNFa, which is critical for blastema formation and priming of caudal fin regeneration in zebrafish ${ }^{12}$. These findings indicate that NCdC could promote the proliferation of blastemal cells indirectly through macrophage activation.

We also observed a substantial upregulation of $n r g 1$ in the regenerating blastema. NRG1 is a critical factor for the development of NC cells and some NCdC, including $\mathrm{SC}^{32}$, that promote the proliferation of damaged tissue cells in various models and during regeneration ${ }^{33,34}$. In zebrafish and mouse, NRG1 promotes and stimulates cardiomyocyte proliferation during heart regeneration and repair, respectively ${ }^{35,39}$ in an ERBB2-dependent manner ${ }^{40,41}$. ErbB2-ErbB3 inhibitors significantly decrease progenitor cell proliferation in the blastema ${ }^{36}$. Nrg1/ErbB2 signalling might play an important role in cardiomyocyte proliferation in the regenerating heart of zebrafish by inducing their metabolic reprogramming ${ }^{42}$. In mammals, NRG1 controls SC proliferation and migration through binding to ErbB2-ErbB3 ${ }^{43}$. Moreover, during cardiac regeneration in adult mice, ERBB2 overexpression in cardiomyocytes promotes an epithelial-mesenchymal-like regenerative response characterized by remodelling of their cytoskeleton, junction dissolution, migration, and extracellular matrix replacement ${ }^{44}$. In line with these studies, we found that erbb2 was upregulated concomitantly with the increased expression of $n r g 1$ in the regenerating blastema. Moreover, exposure to specific ErbB inhibitors significantly impaired blastemal cell proliferation and caudal fin fold regeneration. The finding that $n r g 1$ and erbb2 upregulation in response to fin fold amputation is foxd3-dependent highlights the correlation between nrg1 and the presence of $f o x d 3^{+}$cells in the regenerating blastema. This suggests that $\mathrm{NCdC}$ are required for regeneration through the secretion of NRG1, a paracrine and mitogenic factor. Our data are reminiscent of mammalian digit tip regeneration, where SC precursors secrete paracrine factors, such as oncostatin M (OSM) and PDGF-AA, that enhance mesenchymal cell proliferation and regeneration ${ }^{19}$. Moreover, during mammalian cardiac regeneration, OSM and NRG1 induce cardiomyocyte proliferation and dedifferentiation both in vitro and in vivo $39,45,46$.
As expected from our previous work, we identified macrophages that exert a critical role in the zebrafish caudal fin fold regeneration ${ }^{12}$ among a subgroup of $m p e g 1^{+}$cells in intact and to a greater extent in regenerating fin fold tissues. We then discovered that zebrafish macrophages express erbb2 and erbb3, like mammalian macrophages that express erbb2, erbb3 and migrate in response NRG1 in vitro ${ }^{38}$. These receptors are expressed particularly by $t n \mathrm{fa}^{+}$pro-inflammatory macrophages. Using an ErbB specific inhibitor, we observed a significant decrease in the number of macrophages in the injured fin fold at 6 and $24 \mathrm{hpA}$ associated with a significant reduction of macrophage activation and polarization toward the pro-inflammatory phenotype. This result further confirms NCdC pivotal role during regeneration not only as cells that express mitogenic factors, such as NRG1, but also as cells that induce the release of TNFa by activated proinflammatory macrophages. In the context of nerve injury, NCdC releases cytokines essential for M1-like macrophage recruitment and their polarization toward an anti-inflammatory M2-like macrophage 23 . Moreover, the sequential and well-coordinated recruitment of these two macrophage subsets during appendage regeneration provides the tightly regulated $\mathrm{TNFa}$ signal that orchestrates the process ${ }^{12}$. Consistent with studies describing erbb2, erbb3, and erbb4 expression in macrophages ${ }^{38}$, we elucidated the functional dialogue between macrophage and NCdC during epimorphic regeneration in zebrafish. However, and in contradiction with our results, it was previously shown that the ErbB/NRG1 signalling pathway is not required for immune cell recruitment to the wound in zebrafish caudal fin regeneration ${ }^{36}$. This discrepancy could be explained by the use in this previous study of a transgenic line that is not specific for macrophages or myeloid cells, and by the absence of quantification of the observed effect.

It was previously shown that during zebrafish regeneration, the ErbB/NRG1 signalling pathway ${ }^{36}$ has a role in blastema mesenchymal cell proliferation and migration. However, these authors did not identify the origin of NRG1-producing cells. As the NRG1/ErbB signalling pathway is involved in SC precursor development and biology, the authors used the colourless/sox 10 mutant in which the development of NC derivatives, particularly SC, is altered to study their role in caudal fin regeneration. They did not observe any regeneration impairment in this mutant and concluded that SC are not required for regeneration ${ }^{36}$. This discrepancy with our results could be explained by the fact that foxd3 exhibits a large but incomplete spatiotemporal overlap with sox10 expression ${ }^{47}$. Moreover, the spatiotemporal distribution of the fluorescent proteins in three transgenic lineages, $T g$ (foxd3:GFP), $T g$ (sox10:eGFP), and $T g$ (sox10:mRFP), shows major differences during early NC development ${ }^{36}$. Thus, zebrafish transgenic lines are powerful experimental tools for cell lineage tracing investigations, however, their characterization is critical to address a specific question and provide accurate conclusions. Here, on the basis of previous studies and our results showing the presence of foxd $3^{+}$cells but not of sox $10^{+}$cells in the caudal fin fold mesenchyme, we focused our attention on foxd $3^{+}$ cells to study the role of NC cell derivatives during epimorphic regeneration of the caudal fin fold. Moreover, the transgenic line $T g(f o x d 3: G F P)$ has been used in several studies on NC derivatives, including SC, that also demonstrated the pertinence of focusing on foxd3 to address NCdC role in appendage regeneration ${ }^{48,49}$.

In addition to foxd3 $3^{+}$cells within the regenerating caudal fin fold, we noticed phenotypic and morphological changes of $\mathrm{rcn}^{+}$ caudal fin fold mesenchymal cells that acquired NC-like properties. In line with this result, we observed that while the frequency of $e G F P^{+}$foxd $3^{+} \mathrm{NCdC}$ did not change during the first $24 \mathrm{~h}$ of the regeneration process, the frequency of $m$ Cherry ${ }^{+}$and $m$ Cherry $^{+} e G F P^{+}$mesenchymal cells was significantly increased 
in the regenerating caudal fin fold at $24 \mathrm{hpA}$ compared with the intact fin fold at the same developmental stage. It will be interesting to determine the identity of these mesenchymal cells that express NC markers and their exact function during regeneration. There is a controversy about the origin of mesenchymal cells in the caudal fin in teleost fishes. Kague and colleagues proposed the $\mathrm{NC}$ origin of the caudal fin using genetic-based lineage tracing ${ }^{50}$. Conversely, Lee and colleagues argued that fin mesenchymal cells derive entirely from the mesoderm without any $\mathrm{NC}$ contribution $^{51}$. However, this transition from NCdC toward mesenchymal cells specifically in the regenerating tissue echoes what was observed in the context of mouse digit tip regeneration $^{52}$. Indeed, single-cell profiling showed that mesenchymal blastemal cells are distinct from control digit mesenchymal cells ${ }^{52}$. The authors concluded that within the blastema, cells acquire a mesenchymal transcriptional state and participate in the regeneration of dermis and bone $\mathrm{s}^{52}$. Therefore, we could extrapolate that within the blastema formed after caudal fin fold amputation, NCdC adopt a mesenchymal cell phenotype to contribute to the caudal fin mesenchymal tissue regeneration.

Altogether, these findings demonstrate that foxd $3^{+} \mathrm{NCdC}$ are one of the cell subsets in the regenerating blastema and that they play a major role in epimorphic regeneration in zebrafish. NCdC are required for blastema cell proliferation and blastema formation through the release of the trophic factor NRG1 and the activation of erbb2 $2^{+}$and erbb3 $3^{+}$blastemal macrophages. Activated macrophages express TNFa and thus provide the accurate signal to prime regeneration in zebrafish. This study proposes an integrated view of the regenerative process in a vertebrate in which NCdC activates macrophages and secrete mitogenic paracrine factors. Understanding how the expression of such factors is regulated could be a key to activate lost regeneration processes or to improve the healing response in mammals. Finally, this study underlies the crucial importance of investigating the tight crosstalk between cells during this fine regulated process, which could lead to appropriate therapies for regenerative medicine.

\section{Methods}

Ethics statement. All animal experiments described in this study were carried out at the University of Montpellier according to the European Union guidelines for the handling of laboratory animals (http://ec.europa.eu/environment/chemicals/ lab_animals/home_en.htm) and were approved by the (http://ec.europa.eu/ environment/chemicals/lab_animals/home_en.htm) and were approved by the Comité d'Ethique pour l'Expérimentation Animale under reference CEEA-LR- B4172-37 and APAFIS\#5737-2016061511212601 v3.

Zebrafish lines and maintenance. Embryos were generated from pairs of adult fish by natural spawning and raised in tank water at $28.5^{\circ} \mathrm{C}^{53}$. Experiments were performed using the $\mathrm{AB}$ zebrafish stain (ZIRC), and the transgenic line $\operatorname{Tg}(m p e g 1: m C h e r r y-F)$ to visualize macrophages ${ }^{54}, \mathrm{Tg}($ tnfa:eGFP-F) to visualize tnfa expression $^{17}, \mathrm{Tg}$ (rcn3:gal4/UAS:DsRed) to visualize mesenchymal cells ${ }^{26}, \mathrm{Tg}($ fox$d 3: e G F P-F)^{55}$ and $T g(\text { sox } 10: e G F P-F)^{56}$ to visualize NC cells, $\mathrm{Tg}($ col2a:mCherry) to visualize chondrocytes. Homozygous larvae from the $\operatorname{Tg}($ foxd $3: m C h e r r y)$ ct 110 line were used as Foxd 3 mutants $^{30}$. Embryos were obtained from adult fish pairs by natural spawning and were raised at $28.5^{\circ} \mathrm{C}$ in tank water.

\section{Larva manipulation for regeneration assays and imaging. Caudal fin fold} amputation was performed in $3 \mathrm{dpf}$ larvae under anaesthesia with $0.016 \%$ Tricaine (MS222, Sigma) in zebrafish water using a sterile scalpel ${ }^{57}$. For imaging, live embryos were anesthetized in $0.016 \%$ Tricaine, and positioned in $35 \mathrm{~mm}$ glassbottom dishes (FluoroDish ${ }^{\mathrm{TM}}$, World Precision Instruments). They were mounted in $1 \%$ low melting point agarose (Sigma) with Tricaine. Light microscopy was performed using an MVX10 Olympus macroscope equipped with an MVPLAPO $1 \mathrm{X}$ objective and XC50 camera. Z-stacks series were obtained using an inverted confocal microscope Leica TCS SP5 (Leica Application Suite V3.2) and TCS SP8 (Leica Application Suite V3.5) equipped with an HCXPL APO 40x/1.25-0.75 oil and an HC PL APO $0.70 \infty$ (infinity) 20x objective (Leica). The mCherry signal was excited with a $560 \mathrm{~nm}$ laser, and GFP with a $490 \mathrm{~nm}$ laser. Datasets were analysed using Fiji Software (ImageJ $1.52 \mathrm{p})^{58}$.
Cell isolation, library preparation and data processing of 10x Genomics Chromium scRNA-seq data. Approximately, 150 cut and uncut caudal samples were collected and dissociated into a single-cell suspension. Cell viability and aggregation were tested prior to proceeding with the 10X Genomics protocol for 3' transcript capture and single-cell library preparation. The concentration of freshly dissociated cells was adjusted to $700-800$ cells/ $\mu \mathrm{l}$ in PBS aiming to capture 4000 cells by the $10 \mathrm{X}$ Genomics device. Briefly, the manufacturer's protocol (Chromium ${ }^{\text {in }}$ Single Cell 3' Reagent kit v3.1) was followed to prepare single-cell libraries for Illumina sequencing. Libraries quantification was performed using the Fragment Analyzer system (NGS High Sensitivity Kit) and qPCR (ROCHE, Light Cycler 480). Sequencing was performed in paired-end mode with an S1 flow cell (28/8/87 cycles) using a NovaSeq 6000 sequencer (Illumina) at the MGX core facility of Montpellier. We used cell ranger mkfastq and cellranger count pipelines from the Cell Ranger Single Cell software by 10x Genomics (http://10xgenomics.com) for the initial quality control, sample demultiplexing, mapping, and quantification of raw sequencing data. Quality controls for scRNA-seq are provided (Supplementary Data 5). Raw scRNA-seq data were processed using the Cell Ranger software (Cell Ranger v3.1.0) provided by 10X Genomics with the default options. Briefly, files were first converted to the fastq format, and then sequences were aligned to the Danio rerio reference genome (danRer11) to generate single-cell feature counts using the standard 10x Genomics CellRanger Count pipeline with default parameters. The Cloud software (version 3.1.1) was used to visualize and analyze the results obtained with Cell Ranger. The Uniform Manifold Approximation and Projection (UMAP) dimensional reduction technique was used to visualize data. Output graphing allowed the visualization of cell cluster identity and marker gene expression.

Morpholino injections, Drug treatments. For foxd3 (NM_131290) knock down experiments, morpholino antisense oligonucleotides (Gene Tools) against the ATG site were used (MOfoxd3): 5' TGCTGCTGGAGCAACCCAAGGTAAG 3'. As a control, a Control morpholino $(\mathrm{MOctl})$ from Gene Tools was used: 5' AATCAC AAGCAGTGCAAGCATGATG 3'. Two-three $\mathrm{nl}$ of each morpholino at $500 \mu \mathrm{M}$ concentration was injected in one-cell stage embryos with a Femto. Jet from Eppendorf. No side effect was observed. For Nrgl signalling inhibition, AG1478 (Sigma), and PD168393 (Sigma) were diluted directly in fish water at $10 \mu \mathrm{M}$, and the treatment was renewed every $24 \mathrm{~h}$ after amputation.

In situ hybridization. The plasmid containing junbl (PCRII-junb-l) was kindly sent by Atsushi Kawakami (Department of Biological Information, Tokyo Institute of Technology, Japan), and the plasmid containing $n r g 1$ was kindly sent by Kenneth D. Poss (Department of Cell Biology, Howard Hughes Medical Institute, Duke University Medical Center, Durham, United States). Digoxigenin (DIG)-labelled (Roche) sense and anti-sense RNA probes were prepared using the in vitro Transcription kit (Biolabs). In situ hybridization of whole embryos was performed as detailed in ${ }^{59}$. Embryos were imaged with an Axio Scan from Zeiss with a $40 \mathrm{X}$ 0.95 objective (Zeiss Axio Scan.Z1; Zeiss Axio Imager Z.2).

Cell proliferation detection. For quantification of cell proliferation, whole embryos were fixed in $4 \%$ paraformaldehyde overnight and stained using an antiphosphorylated histone 3 antibody (Cell Signaling, ref 9701, dilution:1/500) ${ }^{12}$.

Larval tail RNA preparation and quantitative RT-PCR. To determine the relative expression of $n r g 1$ type III, type IV, erbb2, erbb3, mpeg1, foxd3, and ef $1 a$, total RNA from larval fin fold (pools of 20 or 30 fin fold) was prepared at 24hpA. Total RNA (20 ng) was reverse-transcribed with the High-Capacity RNA Reverse Transcription kit (Applied Biosystems, France) RT-qPCR analyses were performed using the Light Cycler480 system and the following primers: efla.5(5'-TTCTGTTACCTGG

CAAAGGG-3'), ef1a.3(5'-TTCAGTTTGTCCAACACCCA-3'), erbb2.5(5'-CCATG GCACGGGATCCCTCA-3'), erbb2.3( $5^{\prime}$-GCTGTTGCGCCCACAGGAAG-3'), erb b3.5(5'-GCCCGTGGAGCTCAGAGCATT-3'), erbb3.3(5'-CCAACGGGAAAGGC GCTACTG-3'), nrg1.003.5 (5'-GGCCAGCTTCTACAAAGCTGAGGA-3'), nrg1.0 03.3 (5'-GCTGCAGCGTTTCGCTCTCG-3'), nrg1.004.5 (5'-TGGGATTGAATT TATGGAAGCTGAGGA-3'), nrg1.004.3 (5'-GGTGGAGGGTGAGGGTGTTG-3'), foxd3.5 (5'-CCGGGAGAAGTTTCCGGCCT-3'), foxd3.3 (5'-TGGGGGTCGAGG GTCCAGTA-3'), mpeg.5 (5'-GTGAAAGAGGGTTCTGTTACA-3'), and mpeg.3 (5'-GCCGTAATCAAGTACGAGTT-3').

Monitoring fin fold regeneration, macrophage subset count, cell proliferation, morphological changes and statistical analysis. Caudal fin fold regeneration was monitored by measuring the fin fold growth length from the transected plan (end of the notochord) up to the most proximal end of the fin fold with the Fiji software. Macrophages and cell proliferation in the wound region were measured directly on images acquired by microscopy using the indicated reporter lines and staining. Morphological changes of fox $3^{+} r c n 3^{+}$cells were assessed with the Fiji software using the circularity and roundness plugins. These plugins are an extended version of the ImageJ Measure command that calculates object circularity using the formula: circularity $=4 \mathrm{pi}\left(\right.$ area/perimeter $\left.{ }^{\wedge} 2\right)$. A circularity value of 1.0 indicates a perfect circle. Values approaching 0.0 indicate an increasingly elongated polygon. As roundness $=4$ area $/(\pi$ major_axis $\wedge 2)$, roundness is more relative to the area of the object compared with the main axis. It could be considered as opposite to the elongation factor, whereas circularity refers to the object shape compared to a 
perfect circle. Graphs show the mean \pm standard error of the mean (SEM). The Mann-Whitney test was performed to test the significance of the data presented in all the figures except for Fig. 3a for which Kruskal-Wallis ANOVA with the Dunn's post-hoc test was performed to test the significance of the data using the GraphPad Prism 6 software (San Diego, CA, USA).

Reporting summary. Further information on research design is available in the Nature Research Reporting Summary linked to this article.

\section{Data availability}

All datasets generated in this study have been deposited in the Gene Expression Omnibus repository under the series number GSE158851. Source Data are included with this paper. Source data are provided with this paper.

Received: 7 October 2020; Accepted: 7 September 2021; Published online: 03 November 2021

\section{References}

1. Brockes, J. P. Amphibian limb regeneration: rebuilding a complex structure. Science 276, 81-87 (1997).

2. Gemberling, M., Bailey, T. J., Hyde, D. R. \& Poss, K. D. The zebrafish as a model for complex tissue regeneration. Trends Genet. 29, 611-620 (2013).

3. Knopf, F. et al. Bone regenerates via dedifferentiation of osteoblasts in the zebrafish fin. Developmental Cell 20, 713-724 (2011).

4. Kragl, M. et al. Cells keep a memory of their tissue origin during axolotl limb regeneration. Nature 460, 60-65 (2009).

5. Tornini, V. A. et al. Live monitoring of blastemal cell contributions during appendage regeneration. Curr. Biol. 26, 2981-2991 (2016).

6. Tu, S. \& Johnson, S. L. Fate restriction in the growing and regenerating zebrafish fin. Developmental Cell 20, 725-732 (2011).

7. Johnson, G. L., Masias, E. J. \& Lehoczky, J. A. Cellular heterogeneity and lineage restriction during mouse digit tip regeneration at single-cell resolution. Developmental cell 52, 525-540 e525 (2020).

8. Gerber, T. et al. Single-cell analysis uncovers convergence of cell identities during axolotl limb regeneration. Science 362, https://doi.org/10.1126/ science.aaq0681 (2018).

9. Leigh, N. D. et al. Transcriptomic landscape of the blastema niche in regenerating adult axolotl limbs at single-cell resolution. Nat. Commun. 9, 5153 (2018).

10. Hou, Y. et al. Cellular diversity of the regenerating caudal fin. Sci. Adv. 6, eaba2084 (2020).

11. Petrie, T. A., Strand, N. S., Yang, C. T., Rabinowitz, J. S. \& Moon, R. T. Macrophages modulate adult zebrafish tail fin regeneration. Development 141, 2581-2591 (2014).

12. Nguyen-Chi, M. et al. TNF signaling and macrophages govern fin regeneration in zebrafish larvae. Cell Death Dis. 8, e2979 (2017).

13. Simoes, M. G. et al. Denervation impairs regeneration of amputated zebrafish fins. BMC Developmental Biol. 14, 780 (2014).

14. Godwin, J. W., Pinto, A. R. \& Rosenthal, N. A. Macrophages are required for adult salamander limb regeneration. Proc. Natl Acad. Sci. USA 110, 9415-9420 (2013).

15. Yun, M. H., Davaapil, H. \& Brockes, J. P. Recurrent turnover of senescent cells during regeneration of a complex structure. eLife 4, https://doi.org/10.7554/ eLife.05505 (2015).

16. Petrie, T. A., Strand, N. S., Tsung-Yang, C., Rabinowitz, J. S. \& Moon, R. T. Macrophages modulate adult zebrafish tail fin regeneration. Development 141, 2581-2591 (2014).

17. Nguyen-Chi, M. et al. Identification of polarized macrophage subsets in zebrafish. eLife 4, e07288 (2015).

18. Kumar, A., Godwin, J. W., Gates, P. B., Garza-Garcia, A. A. \& Brockes, J. P. Molecular basis for the nerve dependence of limb regeneration in an adult vertebrate. Science 318, 772-777 (2007).

19. Johnston, A. P. et al. Dedifferentiated Schwann Cell Precursors Secreting Paracrine Factors Are Required for Regeneration of the Mammalian Digit Tip. cell Stem Cell 19, 433-448 (2016).

20. Sande-Melon, M. et al. Adult sox10(+) Cardiomyocytes Contribute to Myocardial Regeneration in the Zebrafish. Cell Rep. 29, 1041-1054 e1045 (2019).

21. Tang, W., Martik, M. L., Li, Y. \& Bronner, M. E. Cardiac neural crest contributes to cardiomyocytes in amniotes and heart regeneration in zebrafish. eLife 8, https://doi.org/10.7554/eLife.47929 (2019).

22. Stratton, J. A. et al. The immunomodulatory properties of adult skin-derived precursor Schwann cells: implications for peripheral nerve injury therapy. Eur. J. Neurosci. 43, 365-375 (2016).

23. Stratton, J. A. \& Shah, P. T. Macrophage polarization in nerve injury: do Schwann cells play a role? Neural Regen. Res. 11, 53-57 (2016).
24. Gammill, L. S. \& Bronner-Fraser, M. Neural crest specification: migrating into genomics. Nat. Rev. Neurosci. 4, 795-805 (2003).

25. Curran, K., Raible, D. W. \& Lister, J. A. Foxd3 controls melanophore specification in the zebrafish neural crest by regulation of Mitf. Developmental Biol. 332, 408-417 (2009).

26. Ellis, K., Bagwell, J. \& Bagnat, M. Notochord vacuoles are lysosome-related organelles that function in axis and spine morphogenesis. J. Cell Biol. 200, 667-679 (2013).

27. Lister, J. A. et al. Zebrafish Foxd3 is required for development of a subset of neural crest derivatives. Developmental Biol. 290, 92-104 (2006).

28. Powell, D. R., Hernandez-Lagunas, L., LaMonica, K. \& Artinger, K. B. Prdmla directly activates foxd 3 and tfap2a during zebrafish neural crest specification. Development 140, 3445-3455 (2013).

29. Stewart, R. A. et al. Zebrafish foxd3 is selectively required for neural crest specification, migration and survival. Developmental Biol. 292, 174-188 (2006).

30. Hochgreb-Hagele, T. \& Bronner, M. E. A novel FoxD3 gene trap line reveals neural crest precursor movement and a role for FoxD3 in their specification. Developmental Biol. 374, 1-11 (2013).

31. Kawakami, A., Fukazawa, T. \& Takeda, H. Early fin primordia of zebrafish larvae regenerate by a similar growth control mechanism with adult regeneration. Developmental Dyn.: Off. Publ. Am. Assoc. Anatomists 231, 693-699 (2004).

32. Liu, X. et al. Specific regulation of NRG1 isoform expression by neuronal activity. J. Neurosci.: Off. J. Soc. Neurosci. 31, 8491-8501 (2011).

33. Farkas, J. E., Freitas, P. D., Bryant, D. M., Whited, J. L. \& Monaghan, J. R. Neuregulin-1 signaling is essential for nerve-dependent axolotl limb regeneration. Development 143, 2724-2731 (2016).

34. Jarde, T. et al. Mesenchymal niche-derived neuregulin-1 drives intestinal stem cell proliferation and regeneration of damaged epithelium. Cell Stem Cell https://doi.org/10.1016/j.stem.2020.06.021 (2020).

35. Shoffner, A., Cigliola, V., Lee, N., Ou, J. \& Poss, K. D. Tp53 suppression promotes cardiomyocyte proliferation during zebrafish heart regeneration. Cell Rep. 32, 108089 (2020).

36. Rojas-Munoz, A. et al. ErbB2 and ErbB3 regulate amputation-induced proliferation and migration during vertebrate regeneration. Developmental Biol. 327, 177-190 (2009).

37. Douglas, M. R. et al. Off-target effects of epidermal growth factor receptor antagonists mediate retinal ganglion cell disinhibited axon growth. Brain 132, 3102-3121 (2009).

38. Fricker, F. R. \& Bennett, D. L. The role of neuregulin-1 in the response to nerve injury. Future Neurol. 6, 809-822 (2011).

39. Bersell, K., Arab, S., Haring, B. \& Kuhn, B. Neuregulin1/ErbB4 signaling induces cardiomyocyte proliferation and repair of heart injury. Cell 138, 257-270 (2009).

40. D'Uva, G. et al. ERBB2 triggers mammalian heart regeneration by promoting cardiomyocyte dedifferentiation and proliferation. Nat. cell Biol. 17, 627-638 (2015).

41. Gemberling, M., Karra, R., Dickson, A. L. \& Poss, K. D. Nrg1 is an injuryinduced cardiomyocyte mitogen for the endogenous heart regeneration program in zebrafish. eLife 4, https://doi.org/10.7554/eLife.05871 (2015).

42. Honkoop, H. et al. Single-cell analysis uncovers that metabolic reprogramming by ErbB2 signaling is essential for cardiomyocyte proliferation in the regenerating heart. eLife 8, https://doi.org/10.7554/eLife.50163 (2019).

43. Newbern, J. \& Birchmeier, C. Nrg1/ErbB signaling networks in Schwann cell development and myelination. Semin. cell developmental Biol. 21, 922-928 (2010).

44. Aharonov A. et al. ERBB2 drives YAP activation and EMT-like processes during cardiac regeneration. bioRxiv preprint https://doi.org/10.1101/ 2020.01.07.897199 (2020)

45. O’Meara, C. C. et al. Transcriptional reversion of cardiac myocyte fate during mammalian cardiac regeneration. Circ. Res. 116, 804-815 (2015).

46. Kubin, $\mathrm{T}$. et al. Oncostatin $\mathrm{M}$ is a major mediator of cardiomyocyte dedifferentiation and remodeling. cell stem cell 9, 420-432 (2011).

47. Dutton, K. A. et al. Zebrafish colourless encodes sox10 and specifies nonectomesenchymal neural crest fates. Development 128, 4113-4125 (2001).

48. Perlin, J. R., Lush, M. E., Stephens, W. Z., Piotrowski, T. \& Talbot, W. S. Neuronal Neuregulin 1 type III directs Schwann cell migration. Development 138, 4639-4648 (2011).

49. Lyons, D. A. et al. erbb3 and erbb2 are essential for schwann cell migration and myelination in zebrafish. Curr. Biol. 15, 513-524 (2005).

50. Kague, E. et al. Skeletogenic fate of zebrafish cranial and trunk neural crest. PloS ONE 7, e47394 (2012).

51. Lee, R. T., Knapik, E. W., Thiery, J. P. \& Carney, T. J. An exclusively mesodermal origin of fin mesenchyme demonstrates that zebrafish trunk neural crest does not generate ectomesenchyme. Development 140, 2923-2932 (2013).

52. Storer, M. A. et al. Acquisition of a unique mesenchymal precursor-like blastema state underlies successful adult mammalian digit tip regeneration. Developmental Cell 52, 509-524.e509 (2020). 
53. Alibaud, L. et al. A Mycobacterium marinum TesA mutant defective for major cell wall-associated lipids is highly attenuated in Dictyostelium discoideum and zebrafish embryos. Mol. Microbiol. 80, 919-934 (2011).

54. Ellett, F., Pase, L., Hayman, J. W., Andrianopoulos, A. \& Lieschke, G. J. mpeg1 promoter transgenes direct macrophage-lineage expression in zebrafish. Blood 117, e49-56 (2011).

55. Gilmour, D. T., Maischein, H. M. \& Nusslein-Volhard, C. Migration and function of a glial subtype in the vertebrate peripheral nervous system. Neuron 34, 577-588 (2002).

56. Carney, T. J. et al. A direct role for Sox 10 in specification of neural crestderived sensory neurons. Development 133, 4619-4630 (2006).

57. Pase, L., Nowell, C. J. \& Lieschke, G. J. In vivo real-time visualization of leukocytes and intracellular hydrogen peroxide levels during a zebrafish acute inflammation assay. Methods Enzymol. 506, 135-156 (2012).

58. Schindelin, J. et al. Fiji: an open-source platform for biological-image analysis. Nat. Methods 9, 676-682 (2012)

59. Nguyen-Chi, M. E. et al. Morphogenesis and cell fate determination within the adaxial cell equivalence group of the zebrafish myotome. PLoS Genet. 8, e1003014 (2012).

\section{Acknowledgements}

This work was supported by Inserm and University of Montpellier. We thank the MRI facility for their assistance, T. Sauka-Spengler (University of Oxford, United Kingdom) for shipping the $\operatorname{Tg}(\text { foxd3-mcherry })^{c t 110}$ zebrafish line, M. Bagnat for shipping Tg(rcn3:gal4/UAS:mCherry) line. A. Kawakami (Tokyo Institute of Technology, Japan) for the junbl plasmid, K. Poss (Duke University Medical Center, USA) for the nrg1 plasmid, R. Kelsh (University of Bath, United Kingdom) for the Fkd6in situ hybridization plasmid. MP and DS acknowledge financial support from the France Génomique National infrastructure, funded as part of "Investissement d'avenir" programme managed by the Agence Nationale pour la Recherche (contract ANR-10-INBS-09). We also thank the Zebrafish facility of the University of Montpellier, the MRI facility for their assistance and the CARTIGEN platform.

\section{Author contributions}

B.L.B. and F.D. designed experiments with input from A.B., A.S., C.B., M.P., D.S., G.T., P.L.C., M.N.C., M.M., and C.J. B.L.B., A.B., C.B., G.T., P.L.C., M.N.C., and M.M. performed experiments, and B.L.B., A.S., M.P., D.S., and M.M. performed the analysis. F.D. and B.L.B. wrote the paper with input from S.A. and C.J.

\section{Competing interests}

The authors declare no competing interests.

\section{Additional information}

Supplementary information The online version contains supplementary material available at https://doi.org/10.1038/s41467-021-26422-5.

Correspondence and requests for materials should be addressed to Farida Djouad.

Peer review information Nature Communications thanks Peng Jiang, James Monaghan, and the other, anonymous, reviewer(s) for their contribution to the peer review of this work. Peer reviewer reports are available.

Reprints and permission information is available at http://www.nature.com/reprints

Publisher's note Springer Nature remains neutral with regard to jurisdictional claims in published maps and institutional affiliations.

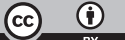

Open Access This article is licensed under a Creative Commons Attribution 4.0 International License, which permits use, sharing, adaptation, distribution and reproduction in any medium or format, as long as you give appropriate credit to the original author(s) and the source, provide a link to the Creative Commons license, and indicate if changes were made. The images or other third party material in this article are included in the article's Creative Commons license, unless indicated otherwise in a credit line to the material. If material is not included in the article's Creative Commons license and your intended use is not permitted by statutory regulation or exceeds the permitted use, you will need to obtain permission directly from the copyright holder. To view a copy of this license, visit http://creativecommons.org/ licenses/by/4.0/.

(C) The Author(s) 2021 